\title{
Quantum scale invariant gravity in the de Donder gauge
}

\author{
Ichiro Oda $\odot^{*}$ \\ Department of Physics, Faculty of Science, University of the Ryukyus, \\ Nishihara, Okinawa 903-0213, Japan
}

(Received 19 January 2022; accepted 17 February 2022; published 2 March 2022)

\begin{abstract}
We perform the manifestly covariant quantization of a scale invariant gravity with a scalar field, which is equivalent to the well-known Brans-Dicke gravity via a field redefinition of the scalar field, in the de Donder gauge condition (or harmonic gauge condition) for general coordinate invariance. First, without specifying the expression of a gravitational theory, we write down various equal-time (anti)commutation relations (ETCRs), in particular, those involving the Nakanishi-Lautrup field, the Faddeev-Popov (FP) ghost, and the FP antighost only on the basis of the de Donder gauge condition. It is shown that choral symmetry, which is a Poincaré-like $\operatorname{IOS} p(8 \mid 8)$ supersymmetry, can be derived from such a general action with the de Donder gauge. Next, taking the scale invariant gravity with a scalar field as a classical theory, we derive the ETCRs for the gravitational sector involving the metric tensor and scalar fields. Moreover, we account for how scale symmetry is spontaneously broken in quantum gravity, thereby showing that the dilaton is a massless Nambu-Goldstone particle.
\end{abstract}

DOI: 10.1103/PhysRevD.105.066001

\section{INTRODUCTION}

A residual symmetry which is left behind after taking a certain gauge-fixing condition for the gauge invariance, has thus far played an important role in quantum field theory. For instance, in string theory, conformal symmetry on the world sheet can be thought as the fundamental symmetry in perturbative regime where strings are weakly interacting [1]. The conformal symmetry is a typical residual symmetry, which is left in a theory after taking the conformal gauge for the world-sheet diffeomorphism [or general coordinate transformation (GCT)] and the Weyl symmetry (or a local scale transformation) [2].

Recently, we have elucidated various aspects of such residual symmetries existing in some gravitational theories. In particular, in the most recent study, we have shown that using the simplest scalar-tensor gravity [3] the restricted Weyl symmetry (RWS) and general coordinate invariance generate conformal symmetry in four dimensions in a flat Minkowski background [4-6].

Also about Einstein gravity, in a pioneering work by Nakanishi $[7,8]$, on the basis of the Einstein-Hilbert action in the de Donder gauge (harmonic gauge) for general coordinate invariance, it has been shown that there remains

\footnotetext{
*ioda@sci.u-ryukyu.ac.jp
}

Published by the American Physical Society under the terms of the Creative Commons Attribution 4.0 International license. Further distribution of this work must maintain attribution to the author(s) and the published article's title, journal citation, and DOI. Funded by SCOAP ${ }^{3}$. a huge residual symmetry, which is a Poincaré-like ISO $p(8 \mid 8)$ supersymmetry, called "choral symmetry," including the BRST symmetry and $G L(4)$ symmetry etc. It is of interest that in this formulation the graviton can be identified with a Nambu-Goldstone particle associated with spontaneous symmetry breakdown of $G L(4)$ symmetry to the Lorentz symmetry $S O(1,3)$, thereby proving the exact masslessness of the graviton in a nonperturbative manner [9].

However, in this formulation, the Einstein equation was critically used in obtaining the equal-time commutation relation $\left[b_{\mu}, \dot{b}_{\nu}^{\prime}\right]=i \tilde{f}\left(\partial_{\mu} b_{\nu}+\partial_{\nu} b_{\mu}\right) \delta^{3}$, which is needed in proving the closure of the $I S O p(8 \mid 8)$ algebra among the generators, so the formulation depends on the EinsteinHilbert term in a classical action. One of our motivations is to relax this situation and show that the choral symmetry does not depend on the expression of the classical gravity but completely comes from the de Donder gauge condition for GCT in the BRST formalism [10]. For this purpose, without the knowledge of the classical Lagrangian we derive various equal-time (anti-)commutation relations (ETCRs) for the Nakanishi-Lautrup field, the FaddeevPopov (FP) ghost, and the FP antighost only on the basis of the de Donder gauge condition.

Another motivation behind the study at hand is to construct a quantum theory of the well-known BransDicke gravity [11] by constructing its manifestly covariant BRST formalism since many of studies of the Brans-Dicke gravity have been limited to a classical analysis. As a concrete advantage of our quantum theory where the gravity as well as a scalar field are quantized, we will 
show that scale invariance is spontaneously broken and consequently "dilaton" is exactly massless thanks to the Nambu-Goldstone theorem even in quantum gravity.

We close this section with an overview of this article. In Sec. II, we discuss a quantum gravity for which the de Donder gauge is adopted as a gauge condition for the general coordinate invariance. In Sec. III, we calculate the ETCRs for the Nakanishi-Lautrup auxiliary field, the FP ghost and the FP antighost based on the quantum gravity made in Sec. II. In Sec. IV, we calculate the ETCRs involving among the Nakanishi-Lautrup field and its time derivative without using the information on a classical gravitational action, and then comment on choral symmetry. In Sec. V, a scale invariant scalar-tensor gravity is briefly reviewed. In Sec. VI, by selecting the scale invariant scalar-tensor gravity as a classical theory we calculate the ETCRs for the gravitational sector, and in Sec. VII we discuss spontaneous symmetry breakdown of the scale invariance and show that the dilaton is exactly massless owing to the Nambu-Goldstone theorem in quantum gravity. The final section is devoted to the conclusion. Two Appendixes are put for technical details. In Appendix A, a derivation of the tensorlike ETCR is given, and in Appendix B we have derived the scale current via the Noether theorem.

\section{QUANTUM GRAVITY WITH DE DONDER GAUGE}

We wish to consider a manifestly covariant canonical formalism of general gravitational theories where general coordinate invariance is fixed by the de Donder gauge condition. To take a more general theory into consideration, without specifying the concrete expression of the gravitational Lagrangian, we will start with the following classical Lagrangian ${ }^{1}$ :

$$
\mathcal{L}_{c}=\mathcal{L}_{c}\left(g_{\mu \nu}, \phi\right),
$$

which includes the metric tensor field $g_{\mu \nu}$ and a scalar field $\phi$ as dynamical fields, and is invariant under the general coordinate transformation (GCT). We assume that $\mathcal{L}_{c}$ does not involve more than first order derivatives of the metric and matter fields.

Let us fix the general coordinate symmetry by the de Donder gauge condition (or harmonic gauge condition):

$$
\partial_{\mu} \tilde{g}^{\mu \nu}=0
$$

\footnotetext{
${ }^{1}$ We follow the notation and conventions of MTW textbook [12]. Greek little letters $\mu, \nu, \cdots$ and Latin ones $i, j, \cdots$ are used for space-time and spatial indices, respectively; for instance, $\mu=0,1,2,3$ and $i=1,2,3$. Furthermore, the Riemann curvature tensor and the Ricci tensor are respectively defined by $R_{\sigma \mu \nu}^{\rho}=\partial_{\mu} \Gamma_{\sigma \nu}^{\rho}+\Gamma_{\lambda \mu}^{\rho} \Gamma_{\sigma \nu}^{\lambda}-(\mu \leftrightarrow \nu)$ and $R_{\mu \nu}=R_{\mu \rho \nu}^{\rho}$.
}

where we have defined $\tilde{g}^{\mu \nu} \equiv \sqrt{-g} g^{\mu \nu} \equiv h g^{\mu \nu}$. Then, the BRST transformation is of the form:

$$
\begin{aligned}
\delta_{B} \bar{c}_{\rho} & =i B_{\rho}, \quad \delta_{B} c^{\rho}=-c^{\lambda} \partial_{\lambda} c^{\rho}, \quad \delta_{B} \phi=-c^{\lambda} \partial_{\lambda} \phi \\
\delta_{B} g_{\mu \nu} & =-\left(\nabla_{\mu} c_{\nu}+\nabla_{\nu} c_{\mu}\right) \\
& =-\left(c^{\alpha} \partial_{\alpha} g_{\mu \nu}+\partial_{\mu} c^{\alpha} g_{\alpha \nu}+\partial_{\nu} c^{\alpha} g_{\mu \alpha}\right), \\
\delta_{B} \tilde{g}^{\mu \nu} & =h\left(\nabla^{\mu} c^{\nu}+\nabla^{\nu} c^{\mu}-g^{\mu \nu} \nabla_{\rho} c^{\rho}\right) .
\end{aligned}
$$

Using this BRST transformation, the Lagrangian for the gauge-fixing condition and FP ghosts can be constructed in a standard manner

$$
\begin{aligned}
\mathcal{L}_{\mathrm{GF}+\mathrm{FP}} & =\delta_{B}\left(i \tilde{g}^{\mu \nu} \partial_{\mu} \bar{c}_{\nu}\right) \\
& =-\tilde{g}^{\mu \nu} \partial_{\mu} B_{\nu}-i \partial_{\mu} \bar{c}_{\nu}\left[\tilde{g}^{\mu \rho} \partial_{\rho} c^{\nu}+\tilde{g}^{\nu \rho} \partial_{\rho} c^{\mu}-\partial_{\rho}\left(\tilde{g}^{\mu \nu} c^{\rho}\right)\right] .
\end{aligned}
$$

To simplify this expression, let us introduce a new auxiliary field $b_{\rho}$ defined as

$$
b_{\rho}=B_{\rho}-i c^{\lambda} \partial_{\lambda} \bar{c}_{\rho}
$$

and its BRST transformation reads

$$
\delta_{B} b_{\rho}=-c^{\lambda} \partial_{\lambda} b_{\rho}
$$

Then, the Lagrangian (4) can be cast to the form:

$\mathcal{L}_{\mathrm{GF}+\mathrm{FP}}=-\tilde{g}^{\mu \nu} \partial_{\mu} b_{\nu}-i \tilde{g}^{\mu \nu} \partial_{\mu} \bar{c}_{\rho} \partial_{\nu} c^{\rho}+i \partial_{\rho}\left(\tilde{g}^{\mu \nu} \partial_{\mu} \bar{c}_{\nu} \cdot c^{\rho}\right)$.

As a result, up to a total derivative, the gauge-fixed and BRST-invariant quantum Lagrangian is given by

$$
\begin{aligned}
\mathcal{L}_{q} & =\mathcal{L}_{c}-\tilde{g}^{\mu \nu} \partial_{\mu} b_{\nu}-i \tilde{g}^{\mu \nu} \partial_{\mu} \bar{c}_{\rho} \partial_{\nu} c^{\rho} \\
& \equiv \mathcal{L}_{c}+\mathcal{L}_{\mathrm{GF}}+\mathcal{L}_{\mathrm{FP}},
\end{aligned}
$$

where we have defined

$$
\mathcal{L}_{\mathrm{GF}} \equiv-\tilde{g}^{\mu \nu} \partial_{\mu} b_{\nu}, \quad \mathcal{L}_{\mathrm{FP}} \equiv-i \tilde{g}^{\mu \nu} \partial_{\mu} \bar{c}_{\rho} \partial_{\nu} c^{\rho} .
$$

By performing the integration by parts once, let us rewrite the Lagrangian (8) as

$$
\begin{aligned}
\mathcal{L}_{q} & =\mathcal{L}_{c}+\partial_{\mu} \tilde{g}^{\mu \nu} b_{\nu}-i \tilde{g}^{\mu \nu} \partial_{\mu} \bar{c}_{\rho} \partial_{\nu} c^{\rho}+\partial_{\mu} \mathcal{V}^{\mu} \\
& \equiv \mathcal{L}_{c}+\overline{\mathcal{L}}_{\mathrm{GF}}+\mathcal{L}_{\mathrm{FP}}+\partial_{\mu} \mathcal{V}^{\mu}
\end{aligned}
$$

where a surface term $\mathcal{V}^{\mu}$ and $\overline{\mathcal{L}}_{\mathrm{GF}}$ are defined as

$$
\mathcal{V}^{\mu} \equiv-\tilde{g}^{\mu \nu} b_{\nu}, \quad \overline{\mathcal{L}}_{\mathrm{GF}} \equiv \partial_{\mu} \tilde{g}^{\mu \nu} b_{\nu}
$$

From this Lagrangian, we can obtain field equations by taking the variation with respect to $g_{\mu \nu}, \phi, b_{\nu}, \bar{c}_{\rho}$, and $c^{\rho}$ in order: 


$$
\begin{aligned}
& \frac{1}{\sqrt{-g}} \frac{\delta \mathcal{L}_{c}}{\delta g^{\mu \nu}}-\frac{1}{2}\left(E_{\mu \nu}-\frac{1}{2} g_{\mu \nu} E\right)=0, \quad \frac{\delta \mathcal{L}_{c}}{\delta \phi}=0, \\
& \partial_{\mu} \tilde{g}^{\mu \nu}=0, \quad g^{\mu \nu} \partial_{\mu} \partial_{\nu} c^{\rho}=0, \quad g^{\mu \nu} \partial_{\mu} \partial_{\nu} \bar{c}_{\rho}=0,
\end{aligned}
$$

where we have defined

$$
\begin{aligned}
E_{\mu \nu} & =\partial_{\mu} b_{\nu}+i \partial_{\mu} \bar{c}_{\rho} \partial_{\nu} c^{\rho}+(\mu \leftrightarrow \nu), \\
E & =g^{\mu \nu} E_{\mu \nu} .
\end{aligned}
$$

Next, in order to find the field equation for the $b_{\rho}$ field, let us take a covariant derivative of the first Einstein equation in Eq. (12). The result reads

$$
\nabla_{\mu}\left(E^{\mu \nu}-\frac{1}{2} g^{\mu \nu} E\right)=0,
$$

where we have used the equation

$$
\nabla^{\mu} \frac{\delta \mathcal{L}_{c}}{\delta g^{\mu \nu}}=0
$$

This equation can be shown as follows: Using the GCT invariance of the classical action, we have

$$
\begin{aligned}
0 & =\delta_{\varepsilon} S_{c} \equiv \int d^{4} x \delta_{\varepsilon} \mathcal{L}_{c}=\int d^{4} x\left(\frac{\delta \mathcal{L}_{c}}{\delta g^{\mu \nu}} \delta_{\varepsilon} g^{\mu \nu}+\frac{\delta \mathcal{L}_{c}}{\delta \phi} \delta_{\varepsilon} \phi\right) \\
& =\int d^{4} x \frac{\delta \mathcal{L}_{c}}{\delta g^{\mu \nu}} \delta_{\varepsilon} g^{\mu \nu}=\int d^{4} x \frac{\delta \mathcal{L}_{c}}{\delta g^{\mu \nu}}\left(\nabla^{\mu} \varepsilon^{\nu}+\nabla^{\nu} \varepsilon^{\mu}\right) \\
& =-2 \int d^{4} x\left(\nabla^{\mu} \frac{\delta \mathcal{L}_{c}}{\delta g^{\mu \nu}}\right) \varepsilon^{\nu}
\end{aligned}
$$

where we have used the field equation for $\phi$.

Generally for a symmetric tensor $S^{\mu \nu}$, we have a formula:

$$
\nabla_{\nu} S_{\mu}^{\nu}=h^{-1} \partial_{\nu}\left(h S_{\mu}^{\nu}\right)+\frac{1}{2} S_{\alpha \beta} \partial_{\mu} g^{\alpha \beta} .
$$

Using this, we can show an equality:

$$
\begin{aligned}
\nabla_{\nu} E^{\nu}{ }_{\mu}= & h^{-1} \partial_{\alpha} \tilde{g}^{\alpha \nu} \cdot E_{\nu \mu}+\partial^{2} b_{\mu} \\
& +i\left(\partial^{2} \bar{c}_{\lambda} \cdot \partial_{\mu} c^{\lambda}+\partial_{\mu} \bar{c}_{\lambda} \cdot \partial^{2} c^{\lambda}\right)+\frac{1}{2} \partial_{\mu} E,
\end{aligned}
$$

where $\partial^{2} \equiv g^{\mu \nu} \partial_{\mu} \partial_{\nu}$. Then Eq. (14), together with the help of the other field equations in Eq. (12), is seen to lead to the field equation for the $b_{\rho}$ field:

$$
g^{\mu \nu} \partial_{\mu} \partial_{\nu} b_{\rho}=0 .
$$

In other words, the $b_{\rho}$ field, the ghost field $c^{\rho}$ and the antighost field $\bar{c}_{\rho}$ all satisfy the d'Alembert equation. Furthermore, it is of interest to see that the space-time coordinates $x^{\lambda}$ obey the d'Alembert equation, $g^{\mu \nu} \partial_{\mu} \partial_{\nu} x^{\lambda}=0$ as well. ${ }^{2}$

\section{EQUAL-TIME COMMUTATION RELATIONS}

In this section, after introducing the canonical commutation relations (CCRs), we will evaluate the equal-time commutation relations (ETCRs) among fundamental variables, in particular, the Nakanishi-Lautrup field $b_{\mu}$, the FP ghost $c^{\mu}$ and the FP antighost $\bar{c}_{\mu}$ in detail. To simplify various expressions, we will obey the following abbreviations adopted in the textbook of Nakanishi and Ojima [8]:

$$
\begin{aligned}
{\left[A, B^{\prime}\right] } & =\left.\left[A(x), B\left(x^{\prime}\right)\right]\right|_{x^{0}=x^{\prime 0}}, \quad \delta^{3}=\delta\left(\vec{x}-\vec{x}^{\prime}\right), \\
\tilde{f} & =\frac{1}{\tilde{g}^{00}}=\frac{1}{\sqrt{-g} g^{00}}=\frac{1}{h g^{00}},
\end{aligned}
$$

where we assume that $\tilde{g}^{00}$ is invertible.

Now let us set up the canonical (anti)commutation relations:

$$
\begin{aligned}
{\left[g_{\mu \nu}, \pi_{g}^{\rho \lambda \prime}\right] } & =i \frac{1}{2}\left(\delta_{\mu}^{\rho} \delta_{\nu}^{\lambda}+\delta_{\mu}^{\lambda} \delta_{\nu}^{\rho}\right) \delta^{3}, \quad\left[\phi, \pi_{\phi}^{\prime}\right]=+i \delta^{3}, \\
\left\{c^{\sigma}, \pi_{c \lambda}^{\prime},\right\} & =\left\{\bar{c}_{\lambda}, \pi_{\bar{c}}^{\sigma \prime}\right\}=+i \delta_{\lambda}^{\sigma} \delta^{3}
\end{aligned}
$$

where the other (anti)commutation relations vanish. Here the canonical variables are $g_{\mu \nu}, \phi, c^{\rho}, \bar{c}_{\rho}$ and the corresponding canonical conjugate momenta are $\pi_{g}^{\mu \nu}, \pi_{\phi}, \pi_{c \rho}, \pi_{\bar{c}}^{\rho}$, respectively and the $b_{\mu}$ field is regarded as not a canonical variable but a conjugate momentum of $\tilde{g}^{0 \mu}$.

Based on the Lagrangian (10), the expressions for canonical conjugate momenta read

$$
\begin{aligned}
\pi_{g}^{\mu \nu} & =\frac{\partial \mathcal{L}}{\partial \dot{g}_{\mu \nu}}, \\
\pi_{\phi} & =\frac{\partial \mathcal{L}}{\partial \dot{\phi}} \\
\pi_{c \sigma} & =\frac{\partial \mathcal{L}}{\partial \dot{c}^{\sigma}}=-i \tilde{g}^{\mu 0} \partial_{\mu} \bar{c}_{\sigma}, \\
\pi_{\bar{c}}^{\sigma} & =\frac{\partial \mathcal{L}}{\partial \dot{\bar{c}}_{\sigma}}=i \tilde{g}^{\mu 0} \partial_{\mu} c^{\sigma},
\end{aligned}
$$

where we have defined the time derivative such as $\dot{g}_{\mu \nu} \equiv \frac{\partial g_{\mu \nu}}{\partial t} \equiv \partial_{0} g_{\mu \nu}$, and differentiation of ghosts is taken from the right.

From now on, we would like to evaluate various nontrivial equal-time commutation relations (ETCRs) in order. Let us first work with the ETCR in Eq. (21):

\footnotetext{
${ }^{2}$ Using the de Donder condition, these d'Alembert equations can be rewritten as $\partial_{\mu}\left(\tilde{g}^{\mu \nu} \partial_{\nu} \Phi\right)=0$ where $\Phi \equiv\left\{x^{\lambda}, b_{\rho}, c^{\sigma}, \bar{c}_{\tau}\right\}$.
} 


$$
\left[\pi_{g}^{\alpha 0}, g_{\mu \nu}^{\prime}\right]=-i \frac{1}{2}\left(\delta_{\mu}^{\alpha} \delta_{\nu}^{0}+\delta_{\mu}^{0} \delta_{\nu}^{\alpha}\right) \delta^{3}
$$

The canonical conjugate momentum $\pi_{g}^{\alpha 0}$ has a structure

$$
\pi_{g}^{\alpha 0}=A^{\alpha}+B^{\alpha \beta} \partial_{\beta} \phi+C^{\alpha \beta} b_{\beta},
$$

where $A^{\alpha}, B^{\alpha \beta}$, and $C^{\alpha \beta} \equiv-\frac{1}{2} \tilde{g}^{00} g^{\alpha \beta}$ have no $\dot{g}_{\mu \nu}$, and $B^{\alpha \beta} \partial_{\beta} \phi$ does not have $\dot{\phi}$ since $\pi_{g}^{\alpha 0}$ does not include the dynamics of the metric and the scalar fields. Then, we find that Eq. (23) produces

$$
\left[g_{\mu \nu}, b_{\rho}^{\prime}\right]=-i \tilde{f}\left(\delta_{\mu}^{0} g_{\rho \nu}+\delta_{\nu}^{0} g_{\rho \mu}\right) \delta^{3} .
$$

From this ETCR, we can easily derive ETCRs:

$$
\begin{aligned}
{\left[g^{\mu \nu}, b_{\rho}^{\prime}\right] } & =i \tilde{f}\left(g^{\mu 0} \delta_{\rho}^{\nu}+g^{\nu 0} \delta_{\rho}^{\mu}\right) \delta^{3}, \\
{\left[\tilde{g}^{\mu \nu}, b_{\rho}^{\prime}\right] } & =i \tilde{f}\left(\tilde{g}^{\mu 0} \delta_{\rho}^{\nu}+\tilde{g}^{\nu 0} \delta_{\rho}^{\mu}-\tilde{g}^{\mu \nu} \delta_{\rho}^{0}\right) \delta^{3}
\end{aligned}
$$

Here we have used the following fact; since a commutator works as a derivation, we can have formulas:

$$
\begin{aligned}
& {\left[g^{\mu \nu}, \Phi^{\prime}\right]=-g^{\mu \alpha} g^{\nu \beta}\left[g_{\alpha \beta}, \Phi^{\prime}\right],} \\
& {\left[\tilde{g}^{\mu \nu}, \Phi^{\prime}\right]=-\left(\tilde{g}^{\mu \alpha} g^{\nu \beta}-\frac{1}{2} \tilde{g}^{\mu \nu} g^{\alpha \beta}\right)\left[g_{\alpha \beta}, \Phi^{\prime}\right],}
\end{aligned}
$$

where $\Phi$ is a generic field.

As for the ETCRs involving ghosts, let us first consider the anti-ETCRs, $\left\{\pi_{c \lambda}, c^{\sigma \prime}\right\}=\left\{\pi_{\bar{c}}^{\sigma}, \bar{c}_{\lambda}^{\prime}\right\}=i \delta_{\lambda}^{\sigma} \delta^{3}$. These ETCRs lead to the same ETCR:

$$
\left\{\dot{\bar{c}}_{\lambda}, c^{\sigma \prime}\right\}=-\tilde{f} \delta_{\lambda}^{\sigma} \delta^{3}
$$

where we have used a useful identity for generic variables $\Phi$ and $\Psi$ :

$$
\left[\Phi, \dot{\Psi}^{\prime}\right]=\partial_{0}\left[\Phi, \Psi^{\prime}\right]-\left[\dot{\Phi}, \Psi^{\prime}\right],
$$

which also holds for the anticommutation relation. Next, it is easy to see that the ETCRs, $\left[\pi_{g}^{\alpha 0}, c^{\sigma^{\prime}}\right]=\left[\pi_{g}^{\alpha 0}, \bar{c}_{\lambda}^{\prime}\right]=0$ produce

$$
\left[b_{\rho}, c^{\sigma^{\prime}}\right]=\left[b_{\rho}, \bar{c}_{\lambda}^{\prime}\right]=0 .
$$

Finally, the ETCRs, $\left[\pi_{c \lambda}, \pi_{g}^{\alpha 0 \prime}\right]=\left[\pi_{\bar{c}}^{\sigma}, \pi_{g}^{\alpha 0 \prime}\right]=0$ give us

$$
\left[\dot{\bar{c}}_{\lambda}, b_{\rho}^{\prime}\right]=-i \tilde{f} \partial_{\rho} \bar{c}_{\lambda} \delta^{3}, \quad\left[\dot{c}^{\sigma}, b_{\rho}^{\prime}\right]=-i \tilde{f} \partial_{\rho} c^{\sigma} \delta^{3} .
$$

In this article, we make use of the following ETCR:

$$
\begin{aligned}
{\left[\dot{g}_{\mu \nu}, b_{\rho}^{\prime}\right]=} & -i\left\{\tilde{f}\left(\partial_{\rho} g_{\mu \nu}+\delta_{\mu}^{0} \dot{g}_{\rho \nu}+\delta_{\nu}^{0} \dot{g}_{\rho \mu}\right) \delta^{3}\right. \\
& \left.+\left[\left(\delta_{\mu}^{k}-2 \delta_{\mu}^{0} \tilde{f} \tilde{g}^{0 k}\right) g_{\rho \nu}+(\mu \leftrightarrow \nu)\right] \partial_{k}\left(\tilde{f} \delta^{3}\right)\right\} .
\end{aligned}
$$

This ETCR can be in general shown to hold when the system has the translational invariance and the general coordinate transformation is fixed by the de Donder gauge as follows: The translational invariance requires the validity of the following equation for a generic field $\Phi(x)$ :

$$
\left[\Phi(x), P_{\rho}\right]=i \partial_{\rho} \Phi(x),
$$

where $P_{\rho}$ is the generator of the translation which is now given by ${ }^{3}$

$$
P_{\rho}=\int d^{3} x \tilde{g}^{0 \lambda} \partial_{\lambda} b_{\rho}
$$

Now let us consider the specific case $\Phi(x)=g_{\mu \nu}(x)$ :

$$
\left[g_{\mu \nu}(x), P_{\rho}\right]=\left[g_{\mu \nu}(x), \quad \int d^{3} x^{\prime} \tilde{g}^{0 \lambda \lambda} \partial_{\lambda} b_{\rho}^{\prime}\right]=i \partial_{\rho} g_{\mu \nu}(x) .
$$

Taking $x^{0}=x^{\prime 0}$ and using $\left[g_{\mu \nu}, \tilde{g}^{0 \lambda \prime}\right]=0$, we have

$$
\int d^{3} x^{\prime} \tilde{g}^{0 \lambda}\left(x^{\prime}\right)\left[g_{\mu \nu}, \partial_{\lambda} b_{\rho}^{\prime}\right]=i \partial_{\rho} g_{\mu \nu}(x)
$$

Using the de Donder gauge condition (2) and Eq. (25), this equation can be rewritten as

$$
\int d^{3} x^{\prime} \tilde{g}^{00}\left(x^{\prime}\right)\left[g_{\mu \nu}, \dot{b}_{\rho}^{\prime}\right]=i\left[\partial_{\rho} g_{\mu \nu}-\frac{1}{\tilde{f}} \partial_{0} \tilde{f}\left(\delta_{\mu}^{0} g_{\rho \nu}+\delta_{\nu}^{0} g_{\rho \mu}\right)\right],
$$

which is easily solved for $\left[g_{\mu \nu}, \dot{b}_{\rho}^{\prime}\right]$ to be

$$
\begin{aligned}
{\left[g_{\mu \nu}, \dot{b}_{\rho}^{\prime}\right]=} & i\left[\tilde{f} \partial_{\rho} g_{\mu \nu}-\partial_{0} \tilde{f}\left(\delta_{\mu}^{0} g_{\rho \nu}+\delta_{\nu}^{0} g_{\rho \mu}\right)\right] \delta^{3} \\
& +F_{(\mu \nu) \rho}{ }^{k} \partial_{k}\left(\tilde{f} \delta^{3}\right)
\end{aligned}
$$

where $F_{(\mu \nu) \rho}{ }^{k}$ is an arbitrary function which is symmetric under the exchange of $\mu \leftrightarrow \nu$.

Next, to fix the function $F_{(\mu \nu) \rho}{ }^{k}$, let us take account of the consistency with the de Donder gauge condition (2):

$$
\left[\partial_{\mu} \tilde{g}^{\mu \nu}, b_{\rho}^{\prime}\right]=0 .
$$

After some calculations, Eq. (39) leads to an equation for $F_{(\mu \nu) \rho}{ }^{k}$ :

$$
\left(\tilde{g}^{0 \alpha} g^{\nu \beta}-\frac{1}{2} \tilde{g}^{0 \nu} g^{\alpha \beta}\right) F_{(\alpha \beta) \rho}{ }^{k}=-i\left(\tilde{g}^{0 k} \delta_{\rho}^{\nu}+\tilde{g}^{0 \nu} \delta_{\rho}^{k}-\tilde{g}^{k \nu} \delta_{\rho}^{0}\right) .
$$

\footnotetext{
${ }^{3}$ This translation generator belongs to the generators of choral symmetry as will be seen in Eq. (62).
} 
This equation has the unique solution given by

$$
F_{(\mu \nu) \rho}^{k}=i\left[\left(\delta_{\mu}^{k}-2 \delta_{\mu}^{0} \tilde{f} \tilde{g}^{0 k}\right) g_{\rho \nu}+(\mu \leftrightarrow \nu)\right] .
$$

We can therefore obtain

$$
\begin{aligned}
{\left[g_{\mu \nu}, \dot{b}_{\rho}^{\prime}\right]=} & i\left\{\left[\tilde{f} \partial_{\rho} g_{\mu \nu}-\partial_{0} \tilde{f}\left(\delta_{\mu}^{0} g_{\rho \nu}+\delta_{\nu}^{0} g_{\rho \mu}\right)\right] \delta^{3}\right. \\
& \left.+\left[\left(\delta_{\mu}^{k}-2 \delta_{\mu}^{0} \tilde{f} \tilde{g}^{0 k}\right) g_{\rho \nu}+(\mu \leftrightarrow \nu)\right] \partial_{k}\left(\tilde{f} \delta^{3}\right)\right\} .
\end{aligned}
$$

Finally, using Eqs. (25) and (29), we can arrive at the desired equation (32). It is of interest that Eq. (32) can be derived from only the translational invariance and the de Donder gauge condition without reference to the classical Lagrangian $\mathcal{L}_{c}$ which knows information of the dynamics of the gravitational field $g_{\mu \nu}$ and the scalar field $\phi$.

Then, using Eq. (32) together with Eqs. (25) and (26), we can easily show that

$$
\begin{aligned}
{\left[\Gamma_{\mu \nu}^{\rho}, b_{\lambda}^{\prime}\right]=} & i \tilde{f}\left(\delta_{\lambda}^{\rho} \Gamma_{\mu \nu}^{0}-\delta_{\mu}^{0} \Gamma_{\lambda \nu}^{\rho}-\delta_{\nu}^{0} \Gamma_{\mu \lambda}^{\rho}\right) \delta^{3} \\
& +i \delta_{\lambda}^{\rho}\left(2 \delta_{\mu}^{0} \delta_{\nu}^{0} \tilde{f} \tilde{g}^{0 k}-\delta_{\mu}^{0} \delta_{\nu}^{k}-\delta_{\nu}^{0} \delta_{\mu}^{k}\right) \partial_{k}\left(\tilde{f} \delta^{3}\right)
\end{aligned}
$$

Incidentally, this ETCR is also needed in deriving the ETCR $\left[b_{\mu}, \dot{b}_{\nu}^{\prime}\right]$ in the next section and the tensorlike ETCR

$$
\left[R_{\mu \nu}, b_{\rho}^{\prime}\right]=-i \tilde{f}\left(\delta_{\mu}^{0} R_{\rho \nu}+\delta_{\nu}^{0} R_{\rho \mu}\right) \delta^{3},
$$

which will be derived in the Appendix A.

Finally, for later convenience we derive the ETCRs, $\left[\phi, b_{\rho}^{\prime}\right],\left[\dot{\phi}, b_{\rho}^{\prime}\right]$ and $\left[\dot{\phi}, \dot{b}_{\rho}^{\prime}\right]$. To do so, we begin by considering $\left[\phi, \bar{c}_{\rho}^{\prime}\right]=0$ and take its BRST transformation as follows ${ }^{4}$ :

$$
\begin{aligned}
0 & =\left\{i Q_{B},\left[\phi, \bar{c}_{\rho}^{\prime}\right]\right\}=\left\{\left[i Q_{B}, \phi\right], \bar{c}_{\rho}^{\prime}\right\}+\left[\phi,\left\{i Q_{B}, \bar{c}_{\rho}^{\prime}\right\}\right] \\
& =\left\{-c^{\lambda} \partial_{\lambda} \phi, \bar{c}_{\rho}^{\prime}\right\}+\left[\phi, i B_{\rho}^{\prime}\right]=\left[\phi, i\left(b_{\rho}^{\prime}+i c^{\lambda \prime} \partial_{\lambda} \bar{c}_{\rho}^{\prime}\right)\right] \\
& =i\left[\phi, b_{\rho}^{\prime}\right]
\end{aligned}
$$

where together with $\left[\dot{\phi}, \bar{c}_{\rho}^{\prime}\right]=0$, Eqs. (3) and (5) have been used. Thus, we have shown

$$
\left[\phi, b_{\rho}^{\prime}\right]=0 .
$$

In a perfectly similar way, we can calculate $\left[\dot{\phi}, b_{\rho}^{\prime}\right]$ by starting with $\left[\dot{\phi}, \bar{c}_{\rho}^{\prime}\right]=0$ as follows:

$$
\begin{aligned}
0 & =\left\{i Q_{B},\left[\dot{\phi}, \bar{c}_{\rho}^{\prime}\right]\right\}=\left\{\left[i Q_{B}, \dot{\phi}\right], \bar{c}_{\rho}^{\prime}\right\}+\left[\dot{\phi},\left\{i Q_{B}, \bar{c}_{\rho}^{\prime}\right\}\right] \\
& =\left\{-\partial_{0}\left(c^{\lambda} \partial_{\lambda} \phi\right), \bar{c}_{\rho}^{\prime}\right\}+\left[\dot{\phi}, i\left(b_{\rho}^{\prime}+i c^{\lambda \prime} \partial_{\lambda} \bar{c}_{\rho}^{\prime}\right)\right] \\
& =-\left\{\dot{c}^{\lambda}, \bar{c}_{\rho}^{\prime}\right\} \partial_{\lambda} \phi+i\left[\dot{\phi}, b_{\rho}^{\prime}\right]
\end{aligned}
$$

\footnotetext{
${ }^{4}$ We define the BRST transformation as $\delta_{B} \Phi \equiv\left[i Q_{B}, \Phi\right\}$ where $\Phi$ is a generic field and [, \} denotes the graded bracket.
}

from which, using Eq. (28) we can obtain

$$
\left[\dot{\phi}, b_{\rho}^{\prime}\right]=-i \tilde{f} \partial_{\rho} \phi \delta^{3} .
$$

The calculation of $\left[\dot{\phi}, \dot{b}_{\rho}^{\prime}\right]$ proceeds as follows: First, we utilize the formula [cf. Eq. (29)]

$$
\left[\dot{\phi}, \dot{b}_{\rho}^{\prime}\right]=\partial_{0}\left(\left[\phi, \dot{b}_{\rho}^{\prime}\right]\right)-\left[\phi, \ddot{b}_{\rho}^{\prime}\right] .
$$

Since the $b$ field obeys the d'Alembert equation as in Eq. (19), $\ddot{b}_{\rho}^{\prime}$ can be described in terms of $\dot{b}_{\rho}$ and $b_{\rho}$ like

$$
\ddot{b}_{\rho}=-\tilde{f}\left(2 \tilde{g}^{0 k} \partial_{k} \dot{b}_{\rho}+\tilde{g}^{k l} \partial_{k} \partial_{l} b_{\rho}\right) .
$$

Then, we are ready to evaluate

$$
\begin{aligned}
{\left[\phi, \ddot{b}_{\rho}^{\prime}\right] } & =-2 \tilde{f}^{\prime} \tilde{g}^{0 k \prime} \partial_{k}^{\prime}\left[\phi, \dot{b}_{\rho}^{\prime}\right]=-2 i \tilde{f}^{\prime} \tilde{g}^{0 k \prime} \partial_{k}^{\prime}\left(\tilde{f} \partial_{\rho} \phi \delta^{3}\right) \\
& =2 i \tilde{f} \partial_{\rho} \phi\left[\tilde{f}^{-1} \partial_{0} \tilde{f} \delta^{3}+\tilde{g}^{0 k} \partial_{k}\left(\tilde{f} \delta^{3}\right)\right] .
\end{aligned}
$$

Thus, we reach a result

$\left[\dot{\phi}, \dot{b}_{\rho}^{\prime}\right]=i \tilde{f}\left(\partial_{\rho} \dot{\phi}-\tilde{f}^{-1} \partial_{0} \tilde{f} \partial_{\rho} \phi\right) \delta^{3}-2 i \tilde{f} \partial_{\rho} \phi \tilde{g}^{0 k} \partial_{k}\left(\tilde{f} \delta^{3}\right)$.

Note that Eqs. (46), (48), and (52) hold as well when we replace $\phi$ by $\varphi$.

\section{DERIVATION OF ETCRS INVOLVING $b_{\mu}$ FIELD AND CHORAL SYMMETRY}

We are now in a position to address the novel part of our formulation and discuss a huge residual symmetry called "choral symmetry" which emerges in adopting the de Donder gauge for the general coordinate invariance.

In order to derive the commutation relations among the $b_{\mu}$ field in terms of the BRST transformation, let us start with the latter equation in Eq. (30) and take the BRST transformation:

$$
\begin{aligned}
0 & =\left\{i Q_{B},\left[b_{\mu}, \bar{c}_{\nu}^{\prime}\right]\right\} \\
& =\left\{\left[i Q_{B}, b_{\mu}\right], \bar{c}_{\nu}^{\prime}\right\}+\left[b_{\mu},\left\{i Q_{B}, \bar{c}_{\nu}^{\prime}\right\}\right] \\
& =-\left\{c^{\rho} \partial_{\rho} b_{\mu}, \bar{c}_{\nu}^{\prime}\right\}+i\left[b_{\mu}, b_{\nu}^{\prime}\right]-\left[b_{\mu}, c^{\rho \prime} \partial_{\rho} \bar{c}_{\nu}^{\prime}\right],
\end{aligned}
$$

where Eqs. (3), (5), and (6) have been used. Using Eq. (31), the first and third terms precisely cancel so we can obtain

$$
\left[b_{\mu}, b_{\nu}^{\prime}\right]=0 .
$$

Next, let us turn our attention to the derivation of the ETCR:

$$
\left[b_{\mu}, \dot{b}_{\nu}^{\prime}\right]=i \tilde{f}\left(\partial_{\mu} b_{\nu}+\partial_{\nu} b_{\mu}\right) \delta^{3}
$$


To do that, let us start with the ETCR

$$
\left[\pi_{c \mu}, b_{\nu}^{\prime}\right]=0,
$$

which can be easily shown. Taking its BRST transformation leads to an equation:

$$
\left[\left\{i Q_{B}, \pi_{c \mu}\right\}, b_{\nu}^{\prime}\right]-\left\{\pi_{c \mu},\left[i Q_{B}, b_{\nu}^{\prime}\right]\right\}=0 .
$$

The first term on the left-hand side (lhs) can be calculated to be

$$
\begin{aligned}
& {\left[\left\{i Q_{B}, \pi_{c \mu}\right\}, b_{\nu}^{\prime}\right]} \\
& \quad=-i\left[\left(\tilde{g}^{\rho \sigma} \nabla_{\sigma} c^{0}+\tilde{g}^{0 \sigma} \nabla_{\sigma} c^{\rho}-\tilde{g}^{\rho 0} \nabla_{\lambda} c^{\lambda}\right) \partial_{\rho} \bar{c}_{\mu}, b_{\nu}^{\prime}\right] \\
& \quad+i \partial_{\nu} b_{\mu} \cdot \delta^{3}-\tilde{g}^{00}\left[b_{\mu}, \dot{b}_{\nu}^{\prime}\right]+i\left[\tilde{g}^{\rho 0} \partial_{\rho}\left(c^{\lambda} \partial_{\lambda} \bar{c}_{\mu}\right), b_{\nu}^{\prime}\right],
\end{aligned}
$$

where Eqs. (3), (5), (22), (26), and (54) have been used. The second term on the lhs in Eq. (57) can be also calculated to be

$$
\left\{\pi_{c \mu},\left[i Q_{B}, b_{\nu}^{\prime}\right]\right\}=-i \partial_{\mu} b_{\nu} \cdot \delta^{3}+c^{\lambda \prime}\left[\pi_{c \mu}, \partial_{\lambda} b_{\nu}^{\prime}\right] .
$$

Then, Eq. (57) together with Eqs. (58) and (59) gives us an equation:

$$
\begin{aligned}
{\left[b_{\mu}, \dot{b}_{\nu}^{\prime}\right]=} & i \tilde{f}\left(\partial_{\mu} b_{\nu}+\partial_{\nu} b_{\mu}\right) \delta^{3} \\
& -i \tilde{f}\left[\left(\tilde{g}^{\rho \sigma} \nabla_{\sigma} c^{0}+\tilde{g}^{0 \sigma} \nabla_{\sigma} c^{\rho}-\tilde{g}^{\rho 0} \nabla_{\lambda} c^{\lambda}\right) \partial_{\rho} \bar{c}_{\mu}, b_{\nu}^{\prime}\right] \\
& +i \tilde{f}\left[\tilde{g}^{\rho 0} \partial_{\rho}\left(c^{\lambda} \partial_{\lambda} \bar{c}_{\mu}\right), b_{\nu}^{\prime}\right]-\tilde{f} c^{\lambda \prime}\left[\pi_{c \mu}, \partial_{\lambda} b_{\nu}^{\prime}\right] \\
\equiv & i \tilde{f}\left(\partial_{\mu} b_{\nu}+\partial_{\nu} b_{\mu}\right) \delta^{3}+K_{\mu \nu} .
\end{aligned}
$$

After some calculations, we can prove $K_{\mu \nu}=0$, which implies that Eq. (55) is certainly valid. Note that in proving $K_{\mu \nu}=0$, it is necessary to make use of Eq. (43) and the field equation for the antighost $\bar{c}_{\mu}$, i.e., $\tilde{g}^{\mu \nu} \partial_{\mu} \partial_{\nu} \bar{c}_{\rho}=0$. In particular, we must use the following ETCRs:

$$
\begin{aligned}
{\left[\ddot{\bar{c}}_{\mu}, b_{\nu}^{\prime}\right]=} & -2 i \tilde{f}\left[\partial_{\nu} \dot{\bar{c}}_{\mu} \delta^{3}-\tilde{g}^{0 k} \partial_{\nu} \bar{c}_{\mu} \partial_{k}\left(\tilde{f} \delta^{3}\right)\right], \\
{\left[\nabla_{\sigma} c^{\rho}, b_{\nu}^{\prime}\right]=} & i \tilde{f}\left(-\delta_{\sigma}^{0} \nabla_{\nu} c^{\rho}+\delta_{\nu}^{\rho} \Gamma_{\sigma \lambda}^{0} c^{\lambda}-\Gamma_{\sigma \nu}^{\rho} c^{0}\right) \delta^{3} \\
& +i \delta_{\nu}^{\rho}\left(2 \delta_{\sigma}^{0} c^{0} \tilde{f} \tilde{g}^{0 k}-\delta_{\sigma}^{0} c^{k}-\delta_{\sigma}^{k} c^{0}\right) \partial_{k}\left(\tilde{f} \delta^{3}\right) .
\end{aligned}
$$

We end this section with the argument of choral symmetry, which is a huge residual symmetry $\operatorname{IOSp}(8 \mid 8)$ involving the BRST symmetry, the rigid translation and $G L(4)$ symmetry etc. Via the Noether theorem, the $\operatorname{IOSp}(8 \mid 8)$ generators can be constructed out of $\mathcal{L}_{\mathrm{GF}}+\mathcal{L}_{\mathrm{FP}}$ in Eq. (9) as [8]

$$
\begin{aligned}
M^{M N} & \equiv \int d^{3} x \tilde{g}^{0 \nu}\left(X^{M} \stackrel{\leftrightarrow}{\partial}_{\nu} X^{N}\right) \\
& \equiv \int d^{3} x \tilde{g}^{0 \nu}\left(X^{M} \partial_{\nu} X^{N}-\partial_{\nu} X^{M} \cdot X^{N}\right), \\
P^{M} & \equiv \int d^{3} x \tilde{g}^{0 \nu}\left(1 \stackrel{\leftrightarrow}{\partial}_{\nu} X^{N}\right) \equiv \int d^{3} x \tilde{g}^{0 \nu} \partial_{\nu} X^{M},
\end{aligned}
$$

where $X^{M} \equiv\left\{x^{\mu}, b_{\mu}, c^{\mu}, \bar{c}_{\mu}\right\}$. The $\operatorname{IOS} p(8 \mid 8)$ algebra takes the graded form:

$$
\begin{aligned}
{\left[i M^{\mathrm{MN}}, M^{\mathrm{RS}}\right\}=} & -M^{\mathrm{MS}} \tilde{\eta}^{\mathrm{NR}}+(-)^{|R||S|} M^{\mathrm{MR}} \tilde{\eta}^{\mathrm{NS}} \\
& -(-)^{|M||N|}(M \leftrightarrow N), \\
{\left[i M^{\mathrm{MN}}, P^{R}\right\}=} & -P^{M} \tilde{\eta}^{\mathrm{NR}}+(-)^{|M||N|} P^{N} \tilde{\eta}^{\mathrm{MR}}, \\
{\left[P^{M}, P^{N}\right\}=} & 0,
\end{aligned}
$$

where $\tilde{\eta}^{\mathrm{MN}}$ is a $16 \times 16 \operatorname{IOSp}(8 \mid 8)$ metric [13].

Since the generators $M^{\mathrm{MN}}$ and $P^{M}$ could have one time derivative, calculating the algebra requires us to use the ETCRs including two time derivatives such as

$$
\begin{aligned}
& {\left[\dot{b}_{\mu}, \dot{b}_{\nu}^{\prime}\right]=i \tilde{f}\left[\partial_{\mu} \dot{b}_{\nu}+\partial_{\nu} \dot{b}_{\mu}-2 \tilde{f} \tilde{g}^{0 \rho} \partial_{\rho} \partial_{\mu} b_{\nu}-\frac{\partial_{0} \tilde{f}}{\tilde{f}}\left(\partial_{\mu} b_{\nu}+\partial_{\nu} b_{\mu}\right)\right] \delta^{3}, \quad-2 i \tilde{f} \tilde{g}^{0 k}\left(\partial_{\mu} b_{\nu}+\partial_{\nu} b_{\mu}\right) \partial_{k}\left(\tilde{f} \delta^{3}\right)} \\
& {\left[\dot{c}^{\mu}, \dot{b}_{\nu}^{\prime}\right]=i \tilde{f}\left[\left(\partial_{\nu} \dot{c}^{\mu}-\frac{\partial_{0} \tilde{f}}{\tilde{f}} \partial_{\nu} c^{\mu}\right) \delta^{3}-2 \tilde{g}^{0 k} \partial_{\nu} c^{\mu} \partial_{k}\left(\tilde{f} \delta^{3}\right)\right],} \\
& {\left[\dot{\bar{c}}_{\mu}, \dot{b}_{\nu}^{\prime}\right]=i \tilde{f}\left[\left(\partial_{\nu} \dot{\bar{c}}_{\mu}-\frac{\partial_{0} \tilde{f}}{\tilde{f}} \partial_{\nu} \bar{c}_{\mu}\right) \delta^{3}-2 \tilde{g}^{0 k} \partial_{\nu} \bar{c}_{\mu} \partial_{k}\left(\tilde{f} \delta^{3}\right)\right] .}
\end{aligned}
$$

These ETCRs can be all derived from the field equations and the ETCRs obtained so far, for instance, Eqs. (12), (19), (29), (30), and (31) without specifying the expression of a classical gravitational Lagrangian. This situation should be contrasted with the previous formulation [8] where Eq. (55) has been derived by using the Einstein-Hilbert Lagrangian. Thus, it is said that the choral symmetry uniquely characterizes the expression of $\mathcal{L}_{\mathrm{GF}}+\mathcal{L}_{\mathrm{FP}}$ [8]. By contrast, we can mention that the origin of the choral symmetry purely lies in the de Donder gauge and the corresponding FP ghost Lagrangian irrespective of a specific choice of a classical gravitational theory as long as there exists the general coordinate invariance in the classical theory. 


\section{A SCALE INVARIANT SCALAR-TENSOR GRAVITY}

In this section, as a classical Lagrangian, we will take the simplest scalar-tensor gravity [3] whose Lagrangian is given by

$$
\mathcal{L}_{c}=\sqrt{-g}\left(\frac{1}{2} \xi \phi^{2} R-\frac{1}{2} g^{\mu \nu} \partial_{\mu} \phi \partial_{\nu} \phi\right)
$$

where $\xi$ is a constant called the nonminimal coupling constant, $\phi$ a real scalar field with a normal kinetic term (i.e., not a ghost), and $R$ the scalar curvature. In addition to the general coordinate transformation (GCT) and a global scale transformation with $\Omega=$ constant, this Lagrangian is also invariant under the restricted Weyl transformation [14-16], [4-6]:

$$
g_{\mu \nu} \rightarrow g_{\mu \nu}^{\prime}=\Omega^{2}(x) g_{\mu \nu}, \quad \phi \rightarrow \phi^{\prime}=\Omega^{-1}(x) \phi,
$$

where the gauge transformation parameter $\Omega(x)$ obeys a constraint $\square \Omega=0$. In order to prove the invariance, we need to use the following transformation of the scalar curvature under (66):

$$
R \rightarrow R^{\prime}=\Omega^{-2}\left(R-6 \Omega^{-1} \square \Omega\right)
$$

For the sake of simplicity, in what follows we will put

$$
\varphi \equiv \frac{1}{2} \xi \phi^{2}
$$

It is worth recalling that we can rewrite (65) as the Lagrangian of the well-known Brans-Dicke theory [11]:

$$
\mathcal{L}_{c}=\sqrt{-g}\left(\varphi R-\omega \frac{1}{\varphi} g^{\mu \nu} \partial_{\mu} \varphi \partial_{\nu} \varphi\right)
$$

where $\omega \equiv \frac{1}{4 \xi}$ is known as the Brans-Dicke parameter. Thus, our classical Lagrangian (65) is at least classically equivalent to that of Brans-Dicke theory.

Taking the de Donder condition as a gauge-fixing condition for GCT, the gauge-fixed and BRST-invariant quantum Lagrangian is given by

$$
\begin{aligned}
\mathcal{L}_{q}= & \sqrt{-g}\left(\varphi R-\frac{1}{2} g^{\mu \nu} \partial_{\mu} \phi \partial_{\nu} \phi\right) \\
& -\tilde{g}^{\mu \nu} \partial_{\mu} b_{\nu}-i \tilde{g}^{\mu \nu} \partial_{\mu} \bar{c}_{\rho} \partial_{\nu} c^{\rho} .
\end{aligned}
$$

From this Lagrangian, we can obtain field equations by taking the variation with respect to $g_{\mu \nu}, \phi, b_{\nu}, \bar{c}_{\rho}$, and $c^{\rho}$ in order: $\varphi G_{\mu \nu}-\left(\nabla_{\mu} \nabla_{\nu}-g_{\mu \nu} \square\right) \varphi-\frac{1}{2} T_{\mu \nu}-\frac{1}{2}\left(E_{\mu \nu}-\frac{1}{2} g_{\mu \nu} E\right)=0$,

$\xi \phi R+\square \phi=0, \quad \partial_{\mu} \tilde{g}^{\mu \nu}=0$,

$g^{\mu \nu} \partial_{\mu} \partial_{\nu} c^{\rho}=0, \quad g^{\mu \nu} \partial_{\mu} \partial_{\nu} \bar{c}_{\rho}=0$,

where we have defined

$$
T_{\mu \nu}=\partial_{\mu} \phi \partial_{\nu} \phi-\frac{1}{2} g_{\mu \nu}\left(\partial_{\rho} \phi\right)^{2} .
$$

Of course, even in this case the $b_{\rho}$ field satisfies the d'Alembert equation (19).

\section{EQUAL-TIME COMMUTATION RELATIONS IN GRAVITATIONAL SECTOR}

Since we have introduced the classical Lagrangian (65) in a theory at hand, we are now ready to evaluate the equaltime commutation relations (ETCRs) involving the metric tensor and the scalar fields in the gravitational sector.

For later convenience, here let us take account of the de Donder gauge condition (2), from which we have identities:

$$
g^{\mu \nu} \Gamma_{\mu \nu}^{\lambda}=0, \quad g^{\lambda \mu} \partial_{\lambda} g_{\mu \nu}=\Gamma_{\lambda \nu}^{\lambda} .
$$

Moreover, since the equation $g^{\mu \nu} \Gamma_{\mu \nu}^{\lambda}=0$ reads

$$
\left(2 g^{\lambda \mu} g^{\nu \rho}-g^{\mu \nu} g^{\lambda \rho}\right) \partial_{\rho} g_{\mu \nu}=0,
$$

it is possible to express the time derivative of the metric field in terms of its spacial one as

$$
\mathcal{D}^{\lambda \mu \nu} \dot{g}_{\mu \nu}=\left(2 g^{\lambda \mu} g^{\nu k}-g^{\mu \nu} g^{\lambda k}\right) \partial_{k} g_{\mu \nu},
$$

where the operator $\mathcal{D}^{\lambda \mu \nu}$ is defined by

$$
\mathcal{D}^{\lambda \mu \nu}=g^{0 \lambda} g^{\mu \nu}-2 g^{\lambda \mu} g^{0 \nu} .
$$

To remove second order derivatives of the metric involved in $R$, we perform the integration by parts once and rewrite the Lagrangian (70) as ${ }^{5}$

$$
\begin{aligned}
\mathcal{L}= & -\varphi \tilde{g}^{\mu \nu}\left(\Gamma_{\mu \nu}^{\sigma} \Gamma_{\sigma \alpha}^{\alpha}-\Gamma_{\mu \alpha}^{\sigma} \Gamma_{\sigma \nu}^{\alpha}\right)-\partial_{\mu} \varphi\left(\tilde{g}^{\alpha \beta} \Gamma_{\alpha \beta}^{\mu}-\tilde{g}^{\mu \nu} \Gamma_{\nu \alpha}^{\alpha}\right) \\
& -\frac{1}{2} \tilde{g}^{\mu \nu} \partial_{\mu} \phi \partial_{\nu} \phi+\partial_{\mu} \tilde{g}^{\mu \nu} b_{\nu}-i \tilde{g}^{\mu \nu} \partial_{\mu} \bar{c}_{\rho} \partial_{\nu} c^{\rho}+\partial_{\mu} \mathcal{V}^{\mu},
\end{aligned}
$$

where a surface term $\mathcal{V}^{\mu}$ is defined as

$$
\mathcal{V}^{\mu}=\varphi\left(\tilde{g}^{\alpha \beta} \Gamma_{\alpha \beta}^{\mu}-\tilde{g}^{\mu \nu} \Gamma_{\nu \alpha}^{\alpha}\right)-\tilde{g}^{\mu \nu} b_{\nu}
$$


From the Lagrangian (77), the concrete expressions for canonical conjugate momenta for the metric tensor and the scalar fields read

$$
\begin{aligned}
\pi_{g}^{\mu \nu}= & \frac{\partial \mathcal{L}}{\partial \dot{g}_{\mu \nu}} \\
= & -\frac{1}{2} \sqrt{-g} \varphi\left[-g^{0 \lambda} g^{\mu \nu} g^{\sigma \tau}-g^{0 \tau} g^{\mu \lambda} g^{\nu \sigma}-g^{0 \sigma} g^{\mu \tau} g^{\nu \lambda}\right. \\
& \left.+g^{0 \lambda} g^{\mu \tau} g^{\nu \sigma}+g^{0 \tau} g^{\mu \nu} g^{\lambda \sigma}+\frac{1}{2}\left(g^{0 \mu} g^{\nu \lambda}+g^{0 \nu} g^{\mu \lambda}\right) g^{\sigma \tau}\right] \partial_{\lambda} g_{\sigma \tau} \\
& -\sqrt{-g}\left[\frac{1}{2}\left(g^{0 \mu} g^{\rho \nu}+g^{0 \nu} g^{\rho \mu}\right)-g^{\mu \nu} g^{\rho 0}\right] \partial_{\rho} \varphi \\
& -\frac{1}{2} \sqrt{-g}\left(g^{0 \mu} g^{\nu \rho}+g^{0 \nu} g^{\mu \rho}-g^{0 \rho} g^{\mu \nu}\right) b_{\rho}, \\
\pi_{\phi}= & \frac{\partial \mathcal{L}}{\partial \dot{\phi}}=-\tilde{g}^{0 \mu} \partial_{\mu} \phi+\xi \phi\left(-\tilde{g}^{\alpha \beta} \Gamma_{\alpha \beta}^{0}+\tilde{g}^{0 \nu} \Gamma_{\nu \alpha}^{\alpha}\right) .
\end{aligned}
$$

From now on, we would like to evaluate several nontrivial equal-time commutation relations (ETCRs) relevant to the gravitational Lagrangian (77). For this purpose, let us first write down some equations to be solved in order. Since $\pi_{\phi}$ in Eq. (79) is rewritten as

$$
\begin{aligned}
\pi_{\phi}= & -\tilde{g}^{00} \dot{\phi}-\tilde{g}^{0 k} \partial_{k} \phi+\xi \phi\left[\left(\tilde{g}^{00} g^{\rho \sigma}-\tilde{g}^{0 \rho} g^{0 \sigma}\right) \dot{g}_{\rho \sigma}\right. \\
& \left.+\left(\tilde{g}^{0 k} g^{\rho \sigma}-\tilde{g}^{0 \rho} g^{k \sigma}\right) \partial_{k} g_{\rho \sigma}\right],
\end{aligned}
$$

$\left[\pi_{\phi}, \phi^{\prime}\right]=-i \delta^{3}$ in Eq. (21) gives rise to an equation:

$-\tilde{g}^{00}\left[\dot{\phi}, \phi^{\prime}\right]+\xi \phi\left(\tilde{g}^{00} g^{\rho \sigma}-\tilde{g}^{0 \rho} g^{0 \sigma}\right)\left[\dot{g}_{\rho \sigma}, \phi^{\prime}\right]=-i \delta^{3}$.

Next, $\left[\pi_{\phi}, g_{\mu \nu}^{\prime}\right]=0$ produces an equation:

$$
-\tilde{g}^{00}\left[\dot{\phi}, g_{\mu \nu}^{\prime}\right]+\xi \phi\left(\tilde{g}^{00} g^{\rho \sigma}-\tilde{g}^{0 \rho} g^{0 \sigma}\right)\left[\dot{g}_{\rho \sigma}, g_{\mu \nu}^{\prime}\right]=0 .
$$

Moreover, $\left[\mathcal{D}^{\lambda \rho \sigma} \dot{g}_{\rho \sigma}, g_{\mu \nu}^{\prime}\right]=0$, which stems from the $\mathcal{D}$-equation (75), reads

$$
\left(g^{0 \lambda} g^{\rho \sigma}-2 g^{\lambda \rho} g^{0 \sigma}\right)\left[\dot{g}_{\rho \sigma}, g_{\mu \nu}^{\prime}\right]=0 .
$$

Similarly, $\left[\mathcal{D}^{\lambda \rho \sigma} \dot{g}_{\rho \sigma}, \phi^{\prime}\right]=0$ gives us an equation:

$$
\left(g^{0 \lambda} g^{\rho \sigma}-2 g^{\lambda \rho} g^{0 \sigma}\right)\left[\dot{g}_{\rho \sigma}, \phi^{\prime}\right]=0 .
$$

Now we are willing to solve Eqs. (81)-(84). First of all, let us focus on Eq. (84). From the argument of symmetry, $\left[\dot{g}_{\rho \sigma}, \phi^{\prime}\right]$ must be of form:

$$
\left[\dot{g}_{\rho \sigma}, \phi^{\prime}\right]=a_{1}\left(g_{\rho \sigma}+a_{2} \delta_{\rho}^{0} \delta_{\sigma}^{0}\right) \delta^{3},
$$

where $a_{1}, a_{2}$ are certain coefficients to be determined sooner. Indeed, from Eq. (84), we find that $a_{2}=\frac{2}{g^{00}}$, so the ETCR (85) reads

$$
\left[\dot{g}_{\rho \sigma}, \phi^{\prime}\right]=a_{1}\left(g_{\rho \sigma}+\frac{2}{g^{00}} \delta_{\rho}^{0} \delta_{\sigma}^{0}\right) \delta^{3}
$$

Next, let us solve Eq. (83). In this case, we also find that $\left[\dot{g}_{\rho \sigma}, g_{\mu \nu}^{\prime}\right]$ has a symmetry under the simultaneous exchange of $(\mu \nu) \leftrightarrow(\rho \sigma)$ and primed $\leftrightarrow$ unprimed in addition to the usual symmetry $\mu \leftrightarrow \nu$ and $\rho \leftrightarrow \sigma$. Then, we can write down its general expression like

$$
\begin{aligned}
{\left[\dot{g}_{\rho \sigma}, g_{\mu \nu}^{\prime}\right]=} & \left\{c_{1} g_{\rho \sigma} g_{\mu \nu}+c_{2}\left(g_{\rho \mu} g_{\sigma \nu}+g_{\rho \nu} g_{\sigma \mu}\right)\right. \\
& +h \tilde{f}\left[c_{3}\left(\delta_{\rho}^{0} \delta_{\sigma}^{0} g_{\mu \nu}+\delta_{\mu}^{0} \delta_{\nu}^{0} g_{\rho \sigma}\right)\right. \\
& \left.+c_{4}\left(\delta_{\rho}^{0} \delta_{\mu}^{0} g_{\sigma \nu}+\delta_{\rho}^{0} \delta_{\nu}^{0} g_{\sigma \mu}+\delta_{\sigma}^{0} \delta_{\mu}^{0} g_{\rho \nu}+\delta_{\sigma}^{0} \delta_{\nu}^{0} g_{\rho \mu}\right)\right] \\
& \left.+(h \tilde{f})^{2} c_{5} \delta_{\rho}^{0} \delta_{\sigma}^{0} \delta_{\mu}^{0} \delta_{\nu}^{0}\right\} \delta^{3}
\end{aligned}
$$

where $c_{i}(i=1, \ldots, 5)$ are some coefficients. Imposing Eq. (83) on (87) leads to relations among the coefficients:

$$
c_{3}=2\left(c_{1}+c_{2}\right), \quad c_{4}=-c_{2}, \quad c_{5}=4\left(c_{1}+c_{2}\right) .
$$

Furthermore, using Eq. (86), Eq. (81) gives $\left[\dot{\phi}, \phi^{\prime}\right]$, which is of form:

$$
\left[\dot{\phi}, \phi^{\prime}\right]=\left(i \tilde{f}+3 a_{1} \xi \phi\right) \delta^{3}
$$

Finally, with the help of Eqs. (86), (87), and (88), Eq. (82) leads to a relation:

$$
a_{1}=\left(3 c_{1}+2 c_{2}\right) \xi \phi \text {. }
$$

In order to fix the coefficients $a_{1}, c_{1}$, and $c_{2}$ completely, we need to have two independent relations among them. Such relations can be provided by calculating $\left[\dot{g}_{k l}, g_{m n}^{\prime}\right]$ explicitly in terms of $\left[\pi_{g}^{k l}, g_{m n}^{\prime}\right]=-i \frac{1}{2}\left(\delta_{m}^{k} \delta_{n}^{l}+\delta_{m}^{l} \delta_{n}^{k}\right) \delta^{3}$ in Eq. (21) and the concrete expression of $\pi_{g}^{k l}$ in Eq. (79). To do that, from Eq. (79), let us write

$$
\pi_{g}^{k l}=\hat{A}^{k l}+\hat{B}^{k l \rho} b_{\rho}+\hat{C}^{k l m n} \dot{g}_{m n}+\hat{D}^{k l} \dot{\varphi} .
$$

Here $\hat{A}^{k l}, \hat{B}^{k l \rho}, \hat{C}^{k l m n}$, and $\hat{D}^{k l}$ commute with $g_{m n}$, and $\hat{C}^{k l m n}$ and $\hat{D}^{k l}$ are defined as ${ }^{6}$

$$
\hat{C}^{k l m n}=\frac{1}{2} h \varphi K^{k l m n}, \quad \hat{D}^{k l}=\tilde{g}^{00} g^{k l}-\tilde{g}^{0 k} g^{0 l},
$$

where the definition of $K^{k l m n}$ and its property are given by

\footnotetext{
${ }^{6}$ It turns out that the concrete expressions of $\hat{A}^{k l}$ and $\hat{B}^{k l \rho}$ are irrelevant to the calculation of $\left[\dot{g}_{k l}, g_{m n}^{\prime}\right]$.
} 


$$
\begin{aligned}
& K^{k l m n}=\left|\begin{array}{ccc}
g^{00} & g^{0 l} & g^{0 n} \\
g^{k 0} & g^{k l} & g^{k n} \\
g^{m 0} & g^{m l} & g^{m n}
\end{array}\right|, \\
& K^{k l m n} \frac{1}{2}\left(g^{00}\right)^{-1}\left(g_{i j} g_{m n}-g_{i m} g_{j n}-g_{\text {in }} g_{j m}\right)=\frac{1}{2}\left(\delta_{i}^{k} \delta_{j}^{l}+\delta_{i}^{l} \delta_{j}^{k}\right) .
\end{aligned}
$$

From Eq. (91), we can calculate

$$
\left[\dot{g}_{k l}, g_{m n}^{\prime}\right]=\hat{C}_{k l p q}^{-1}\left(\left[\pi_{g}^{p q}, g_{m n}^{\prime}\right]-\hat{B}^{p q \rho}\left[b_{\rho}, g_{m n}^{\prime}\right]-\hat{D}^{p q}\left[\dot{\varphi}, g_{m n}^{\prime}\right]\right)
$$

Then, using Eqs. (21), (25), (86), and (93), we find

$$
\begin{aligned}
{\left[\dot{g}_{k l}, g_{m n}^{\prime}\right]=} & \tilde{f} \varphi^{-1}\left[\left(-i-a_{1} \tilde{g}^{00} \xi \phi\right) g_{k l} g_{m n}\right. \\
& \left.+i\left(g_{k m} g_{l n}+g_{k n} g_{l m}\right)\right] \delta^{3} \\
= & {\left[c_{1} g_{k l} g_{m n}+c_{2}\left(g_{k m} g_{l n}+g_{k n} g_{l m}\right)\right] \delta^{3}, }
\end{aligned}
$$

where the last equality comes from Eq. (87). In this way, we have succeeded in getting two independent relations among $a_{1}, c_{1}$, and $c_{2}$ :

$$
c_{1}=\tilde{f} \varphi^{-1}\left(-i-a_{1} \tilde{g}^{00} \xi \phi\right), \quad c_{2}=i \tilde{f} \varphi^{-1} .
$$

Using Eqs. (90) and (96), we can fix completely the coefficients as

$$
c_{1}=-i \frac{4 \xi+1}{6 \xi+1} \tilde{f} \varphi^{-1}, \quad c_{2}=i \tilde{f} \varphi^{-1}, \quad a_{1}=-i \frac{2}{6 \xi+1} \tilde{f} \phi^{-1} .
$$

Accordingly, we can obtain the following ETCRs:

$$
\begin{aligned}
& {\left[\dot{g}_{\rho \sigma}, \phi^{\prime}\right] }=-\frac{2}{6 \xi+1} i \tilde{f} \phi^{-1}\left(g_{\rho \sigma}+\frac{2}{g^{00}} \delta_{\rho}^{0} \delta_{\sigma}^{0}\right) \delta^{3} \\
& {\left[\dot{\phi}, \phi^{\prime}\right]=\frac{1}{6 \xi+1} i \tilde{f} \delta^{3} } \\
& {\left[\dot{g}_{\rho \sigma}, g_{\mu \nu}^{\prime}\right]=} i \tilde{f} \varphi^{-1}\left\{-\frac{4 \xi+1}{6 \xi+1} g_{\rho \sigma} g_{\mu \nu}+g_{\rho \mu} g_{\sigma \nu}+g_{\rho \nu} g_{\sigma \mu}\right. \\
&+h \tilde{f}\left[\frac{4 \xi}{6 \xi+1}\left(\delta_{\rho}^{0} \delta_{\sigma}^{0} g_{\mu \nu}+\delta_{\mu}^{0} \delta_{\nu}^{0} g_{\rho \sigma}\right)\right. \\
&\left.-\left(\delta_{\rho}^{0} \delta_{\mu}^{0} g_{\sigma \nu}+\delta_{\rho}^{0} \delta_{\nu}^{0} g_{\sigma \mu}+\delta_{\sigma}^{0} \delta_{\mu}^{0} g_{\rho \nu}+\delta_{\sigma}^{0} \delta_{\nu}^{0} g_{\rho \mu}\right)\right] \\
&\left.+(h \tilde{f})^{2} \frac{8 \xi}{6 \xi+1} \delta_{\rho}^{0} \delta_{\sigma}^{0} \delta_{\mu}^{0} \delta_{\nu}^{0}\right\} \delta^{3} .
\end{aligned}
$$

It is worthwhile to notice that these ETCRs have two peculiar features, one of which is the presence of the factor
$6 \xi+1$ in the denominator, thereby implying that they do not make sense in a theory with a local scale (or Weyl) symmetry. In other words, in the case of $6 \xi+1=0$ corresponding to the Weyl invariant scalar-tensor gravity, we need to introduce one more gauge condition such as $R=0$ or $\phi=$ constant to fix the Weyl symmetry. The other important feature is the existence of the field $\phi$ (or $\varphi$ ) in the denominator, which means that an unbroken phase $\langle\phi(x)\rangle=0$ cannot be dealt with in the present formalism. This fact might suggest that a (global) scale invariance would be broken spontaneously even in quantum gravity as in classical gravity in order to construct a consistent quantum theory of the scale invariant scalar-tensor gravity. However, there could be the other possibilities. For instance, the present formulation is unable to treat with the symmetric phase. Anyway, we need more study about this problem in the future.

\section{SPONTANEOUS SYMMETRY BREAKDOWN OF SCALE INVARIANCE}

In the previous work, we have shown that the scale invariance is in fact spontaneously broken in classical gravity where the gravitational sector is not quantized [4-6]. In this section, we wish to investigate whether a (global) scale invariance is spontaneously broken even in quantum gravity or not.

Taking $\Omega$ in Eq. (66) to be a constant, we can define a scale transformation as

$$
\begin{aligned}
g_{\mu \nu} & \rightarrow g_{\mu \nu}^{\prime}=\Omega^{2} g_{\mu \nu}, & \phi \rightarrow \phi^{\prime}=\Omega^{-1} \phi, \\
b_{\rho} & \rightarrow b_{\rho}^{\prime}=\Omega^{-2} b_{\rho}, & \bar{c}_{\rho} \rightarrow \bar{c}_{\rho}^{\prime}=\Omega^{-1} \bar{c}_{\rho}, \\
c^{\rho} & \rightarrow c^{\rho \prime}=\Omega^{-1} c^{\rho}, &
\end{aligned}
$$

where we have added the scale transformation for the Nakanishi-Lautrup field and the FP (anti)ghosts. Then, it is easy to see that the quantum Langrangian (70) is invariant under the scale transformation (101). This fact implies that the de Donder gauge is invariant under the scale transformation. Incidentally, the de Donder gauge is not invariant under a local scale (or Weyl) transformation. To make a gauge condition for the general coordinate invariance be invariant under the Weyl transformation requires us to take a different gauge condition such as $\partial_{\mu}\left((-g)^{\frac{1}{4}} g^{\mu \nu}\right)=0$ or $\partial_{\mu}\left(\sqrt{-g} \phi^{2} g^{\mu \nu}\right)=0$ from the de Donder gauge.

Since the scale transformation is a global one, we can construct a conserved Noether current and charge along the standard procedure. After some calculations, it turns out that the conserved current for the scale symmetry is given by ${ }^{7}$

\footnotetext{
${ }^{7}$ See Appendix B.
} 


$$
J^{\mu}=\tilde{g}^{\mu \nu}\left[\frac{6 \xi+1}{2} \partial_{\nu}\left(\phi^{2}\right)+2 b_{\nu}+i \partial_{\nu}\left(\bar{c}_{\rho} c^{\rho}\right)\right] .
$$

It is straightforward to verify that this current is conserved, $\partial_{\mu} J^{\mu}=0$, in terms of field equations. Note that in the conformal coupling $\xi=-\frac{1}{6}$, the first term on the right-hand side (rhs) coming from the classical action is identically vanishing $[17,18]$ while the second and third terms coming from the gauge-fixing and FP ghost terms, respectively, do not so.

The Noether charge $Q=\int d^{3} x J^{0}$ turns out to generate the infinitesimal scale transformation correctly by using the ETCRs obtained thus far:

$$
\begin{aligned}
& \delta g_{\mu \nu}=2 \Lambda g_{\mu \nu}, \quad \delta \phi=-\Lambda \phi, \quad \delta b_{\rho}=-2 \Lambda b_{\rho}, \\
& \delta \bar{c}_{\rho}=-\Lambda \bar{c}_{\rho}, \quad \delta c^{\rho}=-\Lambda c^{\rho},
\end{aligned}
$$

where we have set $\Omega=e^{\Lambda} \approx 1+\Lambda$. It might be curious about why no derivative of the metric tensor $g_{\mu \nu}$ appears in $Q$ since it usually generates the transformation of the metric tensor [3]. This problem can be understood for the first time in the present formulation since we have successfully quantized the metric field and the scalar field.

To check that the charge $Q$ indeed generates the scale transformation (103), let us calculate $\delta g_{\mu \nu}$ and $\delta b_{\rho}$ explicitly. As for $\delta g_{\mu \nu}$,

$$
\begin{aligned}
\delta g_{\mu \nu} & \equiv\left[i \Lambda Q, g_{\mu \nu}\right] \\
& =i \Lambda \int d^{3} x^{\prime}\left[\tilde{g}^{0 \rho \prime}\left(\frac{6 \xi+1}{2} \partial_{\rho}\left(\phi^{\prime 2}\right)+2 b_{\rho}^{\prime}+i \partial_{\rho}\left(\bar{c}_{\sigma}^{\prime} c^{\sigma \prime}\right)\right), g_{\mu \nu}\right] \\
& =i \Lambda \int d^{3} x^{\prime}\left\{(6 \xi+1) \tilde{g}^{00 \prime} \phi^{\prime}\left[\dot{\phi}^{\prime}, g_{\mu \nu}\right]+2 \tilde{g}^{0 \rho \prime}\left[b_{\rho}^{\prime}, g_{\mu \nu}\right]\right\} \\
& =2 \Lambda g_{\mu \nu},
\end{aligned}
$$

where in the third equality we put $x^{0}=x^{\prime 0}$, and used Eqs. (25) and (98). Against the expectation that the derivative of $\phi$ would play a role [3], the $b$ field also does the job in generating the scale transformation. In a similar manner, as for $\delta b_{\rho}$, we have

$$
\begin{aligned}
\delta b_{\rho} & \equiv\left[i \Lambda Q, b_{\rho}\right] \\
& =i \Lambda \int d^{3} x^{\prime}\left[\tilde{g}^{0 \nu \prime}\left(\frac{6 \xi+1}{2} \partial_{\nu}\left(\phi^{\prime 2}\right)+2 b_{\nu}^{\prime}+i \partial_{\nu}\left(\bar{c}_{\sigma}^{\prime} c^{\sigma \prime}\right)\right), b_{\rho}\right] \\
& =i \Lambda \int d^{3} x^{\prime}\left\{\left[\tilde{g}^{0 \nu \prime}, b_{\rho}\right]\left(\frac{6 \xi+1}{2} \partial_{\nu}\left(\phi^{\prime 2}\right)+2 b_{\nu}^{\prime}+i \partial_{\nu}\left(\bar{c}_{\sigma}^{\prime} c^{\sigma \prime}\right)\right)+\tilde{g}^{00 \prime}\left((6 \xi+1) \phi^{\prime}\left[\dot{\phi}^{\prime}, b_{\rho}\right]+i\left(\left[\dot{\bar{c}}_{\sigma}^{\prime}, b_{\rho}\right] c^{\sigma \prime}+\bar{c}_{\sigma}^{\prime}\left[\dot{c}^{\sigma \prime}, b_{\rho}\right]\right)\right)\right\} \\
& =-2 \Lambda b_{\rho},
\end{aligned}
$$

where we have used Eqs. (26), (31), and (48). We wish to mention again that in the case of classical gravity, one cannot show that the charge $Q$ generates the scale transformation owing to the absence of the ETCRs relevant to the Nakanishi-Lautrup field $b_{\rho}$ and the FP ghosts $\bar{c}_{\sigma}$ and $c^{\sigma}$.

Now let us move to the issue of spontaneous symmetry breakdown of scale symmetry in quantum gravity. From Eq. (103) and the definition of $\delta \Phi \equiv[i \Lambda Q, \Phi]$ for a generic field $\Phi$, we obtain that

$$
\begin{aligned}
& {\left[i Q, g_{\mu \nu}\right]=2 g_{\mu \nu}, \quad[i Q, \phi]=-\phi, \quad\left[i Q, b_{\rho}\right]=-2 b_{\rho} \text {, }} \\
& {\left[i Q, \bar{c}_{\rho}\right]=-\bar{c}_{\rho}, \quad\left[i Q, c^{\rho}\right]=-c^{\rho} .}
\end{aligned}
$$

Assuming that the fields take the following vacuum expectation values:

$$
\begin{aligned}
\left\langle 0\left|g_{\mu \nu}\right| 0\right\rangle & =\eta_{\mu \nu}, \quad\langle 0|\phi| 0\rangle=\phi_{0}, \quad\left\langle 0\left|b_{\rho}\right| 0\right\rangle=0, \\
\left\langle 0\left|\bar{c}_{\rho}\right| 0\right\rangle & =0, \quad\left\langle 0\left|c^{\rho}\right| 0\right\rangle=0,
\end{aligned}
$$

with $\phi_{0}$ being a constant, Eq. (106) implies that

$$
\begin{aligned}
\left\langle 0\left|\left[i Q, g_{\mu \nu}\right]\right| 0\right\rangle & =2 \eta_{\mu \nu}, \quad\langle 0|[i Q, \phi]| 0\rangle=-\phi_{0}, \\
\left\langle 0\left|\left[i Q, b_{\rho}\right]\right| 0\right\rangle & =0, \\
\left\langle 0\left|\left[i Q, \bar{c}_{\rho}\right]\right| 0\right\rangle & =0, \quad\left\langle 0\left|\left[i Q, c^{\rho}\right]\right| 0\right\rangle=0 .
\end{aligned}
$$

The second equation in Eq. (108) shows that the scale invariance is spontaneously broken at the quantum level as long as $\phi_{0} \neq 0$ holds $[4,6]$.

Here three important remarks are in order. First, the first assumption in Eq. (107), $\left\langle 0\left|g_{\mu \nu}\right| 0\right\rangle=\eta_{\mu \nu}$, comes from our postulate that the vacuum is invariant under translation [8]:

$$
P_{\mu}|0\rangle=0,
$$


which means that translational invariance is not broken spontaneously. It is true that the existence of the nonvanishing cosmological constant is not consistent with this assumption, but we have adopted this assumption from phenomenological considerations. Moreover, with this assumption the graviton can be identified with a NambuGoldstone (NG) boson corresponding to spontaneous symmetry breakdown of $G L(4)$ symmetry down to $S O(1,3)$ Lorentz symmetry, thereby proving the exact masslessness of the graviton [9].

Second, let us pay our attention to the second assumption in Eq. (107), $\langle 0|\phi| 0\rangle=\phi_{0} \neq 0$, which is also physically plausible by the following argument: As usual, let us consider to move from the Jordan frame to the Einstein frame by implementing a local scale transformation only for the metric tensor field as

$$
g_{\mu \nu} \rightarrow g_{* \mu \nu}=\Omega(x)^{2} g_{\mu \nu}=\frac{1}{M_{\mathrm{Pl}}^{2}} \xi \phi^{2} g_{\mu \nu},
$$

where $M_{\mathrm{Pl}}$ is the reduced Planck mass. Then, in the Einstein frame, up to a surface term the quantum Lagrangian (70) is reduced to the form:

$$
\begin{aligned}
\mathcal{L}_{q}= & \sqrt{-g_{*}}\left(\frac{M_{\mathrm{Pl}}^{2}}{2} R_{*}-\frac{1}{2} g_{*}^{\mu \nu} \partial_{\mu} \sigma \partial_{\nu} \sigma\right) \\
& -e^{-\frac{2 \zeta}{M_{\mathrm{Pl}}} \sigma}\left(\tilde{g}_{*}^{\mu \nu} \partial_{\mu} b_{\nu}-i \tilde{g}_{*}^{\mu \nu} \partial_{\mu} \bar{c}_{\rho} \partial_{\nu} c^{\rho}\right),
\end{aligned}
$$

where we have defined a scalar field $\sigma(x)$ and a constant $\zeta$ as

$$
\phi=\xi^{-\frac{1}{2}} M_{\mathrm{Pl}} e^{\frac{\zeta}{M_{\mathrm{Pl}}} \sigma}, \quad \zeta=\sqrt{\frac{\xi}{6 \xi+1}} .
$$

In this way, we can arrive at the Lagrangian in the Einstein frame by starting with that in the Jordan frame. The key point for the change of the frames lies in Eq. (110) where the scale factor $\Omega(x)$ is proportional to $\frac{1}{M_{\mathrm{Pl}}} \phi(x)$. Namely, the existence of the nonvanishing "dilaton" $\phi \neq 0$, or more precisely, $\langle 0|\phi| 0\rangle \neq 0$, makes it possible to move from the Jordan frame to the Einstein one. In this sense, our assumption $\langle 0|\phi| 0\rangle=\phi_{0} \neq 0$ makes sense physically.

As a final remark, as mentioned in the previous section, in order to make a consistent quantum gravity based on the scale invariant scalar-tensor gravity, it is necessary to require the condition $\langle 0|\phi| 0\rangle \neq 0$. Any physical theories must be formulated within the framework of quantum field theories, so it is natural to assume such the condition.

To close this section, let us verify more explicitly that the spontaneous symmetry breakdown of the scale symmetry occurs in the Einstein frame where the scale symmetry is replaced with a shift symmetry. For this purpose, let us rewrite the charge for the scale symmetry in the Jordan frame into that in the Einstein frame as
$Q=\int d^{3} x \tilde{g}_{*}^{0 \nu}\left[\frac{M_{\mathrm{Pl}}}{\zeta} \partial_{\nu} \sigma+e^{-\frac{2 \zeta}{M_{\mathrm{Pl}}} \sigma}\left(2 b_{\nu}+\partial_{\nu}\left(\bar{c}_{\rho} c^{\rho}\right)\right)\right]$,

where we have used Eqs. (102), (110), and (112). Since $Q$ has a linear term in $\sigma(x)$, the charge cannot annihilate the vacuum $|0\rangle$ :

$$
Q|0\rangle \neq 0
$$

which means the spontaneous symmetry breakdown of scale symmetry in the Jordan frame or shift symmetry in the Einstein frame. Actually, from the Lagrangian (111) the canonical conjugate momentum for the scalar field $\sigma(x)$ reads

$$
\pi_{\sigma} \equiv \frac{\partial \mathcal{L}}{\partial \partial_{0} \sigma}=-\sqrt{-g_{*}} g_{*}^{0 \nu} \partial_{\nu} \sigma .
$$

Then, $Q$ can be rewritten as

$$
Q=-\frac{M_{\mathrm{Pl}}}{\zeta} \int d^{3} x \pi_{\sigma}+\cdots,
$$

where ... denote contributions from the Nakanishi-Lautrup field and the FP ghosts. Using the equal-time commutation relation $\left[\sigma, \pi_{\sigma}^{\prime}\right]=i \delta^{3}$, we obtain

$$
[i Q, \sigma(x)]=-\frac{M_{\mathrm{Pl}}}{\zeta} .
$$

Taking the vacuum expectation value of this equation yields

$$
\langle 0|[i Q, \sigma(x)]| 0\rangle=-\frac{M_{\mathrm{Pl}}}{\zeta} \neq 0,
$$

which clearly means the spontaneous symmetry breakdown and the scalar field $\sigma(x)$ is the massless NG boson for the shift symmetry.

\section{CONCLUSIONS}

In this article, we have performed a manifestly covariant quantization of a scale invariant gravity which is equivalent to the Brans-Dicke gravity [11] via the field redefinition of a scalar field. Many of studies of the Brans-Dicke gravity have been thus far confined to the classical analysis, so it is expected that our quantum formulation of the Brans-Dicke gravity could provide us with some useful information on quantum aspects of the theory.

Actually, we have presented two new results, one of which is that in classically scale invariant gravitational theories, we have a quantum scale symmetry in addition to a huge choral symmetry when we choose the de Donder gauge for the general coordinate invariance. In this respect, it is worthwhile to recall that in the manifestly scale invariant regularization method [19-24], the scale invariance is free of scale anomaly. 
As the second result, we have shown that the scale symmetry is spontaneously broken by quantum effects, thus proving that the "dilaton" is exactly massless because of the Nambu-Goldstone theorem. As pointed out in the previous paper [25], when the dilaton is exactly massless at the quantum level, it mediates a long-range force between massive objects as in the Newtonian force, which imposes a severe phenomenological constraint on parameters in the Brans-Dicke model [11] since the long-range force stemming from the massless dilaton could affect the perihelion advance of Mercury, for instance.

Furthermore, we have shown that the choral symmetry, which is a Poincaré-like $\operatorname{IOS} p(8 \mid 8)$ supersymmetry, can be derived from any gravitational theories, which are invariant under the general coordinate transformation (GCT), if the GCT is gauge-fixed by the de Donder gauge. To put it differently, the choral symmetry comes from only the de Donder gauge for the GCT in the BRST formalism. It is worthwhile to recall that the choral symmetry includes the BRST symmetry and the physical observables can be defined as operators which commute with the BRST charge [8].

We still have a lot of work to be done in the future. For instance, we would like to extend the present formalism to gravitational theories with a local scale invariance (or Weyl invariance) and investigate the resultant residual symmetries. In the case of the Weyl invariance, it seems that we might prefer the Weyl-invariant gauge conditions such as $\partial_{\mu}\left((-g)^{\frac{1}{4}} g^{\mu \nu}\right)=0$ and $\partial_{\mu}\left(\sqrt{-g} \phi^{2} g^{\mu \nu}\right)=0$ to the de Donder gauge condition $\partial_{\mu}\left(\sqrt{-g} g^{\mu \nu}\right)=0$ since we have the restricted Weyl symmetry in these Weyl invariant gauge conditions when we take the gauge condition $R=0$ for the Weyl invariance. However, then we will lose the choral symmetry but instead we would have new residual symmetries.

As another problem, it is of interest to construct a manifestly scale-invariant regularization scheme in the theory at hand and calculate an effective potential explicitly [26-28]. With this regularization scheme, it is necessary to introduce an additional scalar field in addition to the dilaton and the two scalar fields might collaborate with each other for nullifying the scalar force. In fact, such an approach on the basis of the dilaton and the axion has been recently proposed [29]. In the near future, we would like to report on these problems.

\section{ACKNOWLEDGMENTS}

We are grateful to T. Kugo for valuable discussions, in particular, on Secs. II and III. This work is partly supported by the JSPS Kakenhi Grant No. 21K03539.

\section{APPENDIX A: DERIVATION OF $\left[\boldsymbol{R}_{\mu \nu}, b_{\rho}^{\prime}\right]$}

In this Appendix, we wish to prove the tensorlike ETCR:

$$
\left[R_{\mu \nu}, b_{\rho}^{\prime}\right]=-i \tilde{f}\left(\delta_{\mu}^{0} R_{\rho \nu}+\delta_{\nu}^{0} R_{\rho \mu}\right) \delta^{3} .
$$

Our strategy for the proof is similar to that of [8], but is in essence different from it since we have been already able to derive the ETCR (55) without recourse to the Einstein equation. In what follows, we will prove the following two equations:

$\left[G^{0}{ }_{\nu}, b_{\rho}^{\prime}\right]=i \tilde{f}\left(\delta_{\rho}^{0} R_{\nu}^{0}-\delta_{\nu}^{0} R_{\rho}^{0}\right) \delta^{3}, \quad\left[R_{k l}, b_{\rho}^{\prime}\right]=0$,

from which it is easy to see that we can reach our goal (A1).

Let us first prove the former equation in (A2). From the Einstein equation, which is the first equation in (71), $G^{0}{ }_{\nu}$ is

$G_{\nu}^{0}=\frac{1}{\varphi}\left[\left(\nabla^{0} \nabla_{\nu}-\delta_{\nu}^{0} \square\right) \varphi+\frac{1}{2} T^{0}{ }_{\nu}+\frac{1}{2}\left(E^{0}{ }_{\nu}-\frac{1}{2} \delta_{\nu}^{0} E\right)\right]$.

Note that the first term $\left(\nabla^{0} \nabla_{\nu}-\delta_{\nu}^{0} \square\right) \varphi$ contains no $\ddot{\varphi}$. Then, $\left[G_{\nu}^{0}, b_{\rho}^{\prime}\right]$ reads

$$
\begin{aligned}
{\left[G_{\nu}^{0}, b_{\rho}^{\prime}\right]=} & \frac{1}{\varphi}\left\{\left[\left(\nabla^{0} \nabla_{\nu}-\delta_{\nu}^{0} \square\right) \varphi, b_{\rho}^{\prime}\right]+\frac{1}{2}\left[T^{0}{ }_{\nu}, b_{\rho}^{\prime}\right]\right. \\
& \left.+\frac{1}{2}\left[E^{0}{ }_{\nu}-\frac{1}{2} \delta_{\nu}^{0} E, b_{\rho}^{\prime}\right]\right\} \\
\equiv & \frac{1}{\varphi}\left(A_{1}+A_{2}+A_{3}\right) .
\end{aligned}
$$

After some calculations, $A_{1}$ is found to be

$$
\begin{aligned}
A_{1} & \equiv\left[\left(\nabla^{0} \nabla_{\nu}-\delta_{\nu}^{0} \square\right) \varphi, b_{\rho}^{\prime}\right] \\
& =i \tilde{f}\left(\delta_{\rho}^{0} \nabla^{0} \nabla_{\nu} \varphi-\delta_{\nu}^{0} \nabla^{0} \nabla_{\rho} \varphi\right) \delta^{3} .
\end{aligned}
$$

In order to evaluate $A_{2}$, it is necessary to calculate $\left[T_{\mu \nu}, b_{\rho}^{\prime}\right]$ whose result is given by

$$
\left[T_{\mu \nu}, b_{\rho}^{\prime}\right]=-i \tilde{f}\left(\delta_{\mu}^{0} T_{\rho \nu}+\delta_{\nu}^{0} T_{\rho \mu}\right) \delta^{3} .
$$

Using this result, $A_{2}$ becomes

$$
A_{2} \equiv \frac{1}{2}\left[T^{0}{ }_{\nu}, b_{\rho}^{\prime}\right]=\frac{1}{2} i \tilde{f}\left(\delta_{\rho}^{0} T^{0}{ }_{\nu}-\delta_{\nu}^{0} T^{0}{ }_{\rho}\right) \delta^{3} .
$$

Finally, evaluating $A_{3}$ requires us to calculate $\left[E_{\mu \nu}, b_{\rho}^{\prime}\right]$, which is found to be

$$
\left[E_{\mu \nu}, b_{\rho}^{\prime}\right]=-i \tilde{f}\left(\delta_{\mu}^{0} E_{\rho \nu}+\delta_{\nu}^{0} E_{\rho \mu}\right) \delta^{3} .
$$

Since we can calculate $\left[E, b_{\rho}^{\prime}\right]=0, A_{3}$ reads

$$
A_{3} \equiv \frac{1}{2}\left[E^{0}{ }_{\nu}-\frac{1}{2} \delta_{\nu}^{0} E, b_{\rho}^{\prime}\right]=\frac{1}{2} i \tilde{f}\left(\delta_{\rho}^{0} E^{0}{ }_{\nu}-\delta_{\nu}^{0} E_{\rho}^{0}\right) \delta^{3} .
$$

Adding $A_{1}, A_{2}$, and $A_{3}$ gives rise to the former equation in (A2) since from Eq. (71) $R^{0}{ }_{\nu}$ is described as 
$R_{\nu}^{0}=\frac{1}{\varphi}\left[\left(\nabla^{0} \nabla_{\nu}+\frac{1}{2} \delta_{\nu}^{0} \square\right) \varphi+\frac{1}{2}\left(T^{0}{ }_{\nu}-\frac{1}{2} \delta_{\nu}^{0} T\right)+\frac{1}{2} E^{0}{ }_{\nu}\right]$.

Next, let us prove the validity of the latter equation in (A2). From the Einstein equation in (71), $R_{k l}$ takes the form:

$$
R_{k l}=\frac{1}{\varphi}\left[\left(\nabla_{k} \nabla_{l}+\frac{1}{2} g_{k l} \square\right) \varphi+\frac{1}{2}\left(T_{k l}-\frac{1}{2} g_{k l} T\right)+\frac{1}{2} E_{k l}\right] .
$$

With the help of Eqs. (A6) and (A8), we find

$$
\left[R_{k l}, b_{\rho}^{\prime}\right]=\frac{1}{\varphi}\left[\left(\nabla_{k} \nabla_{l}+\frac{1}{2} g_{k l} \square\right) \varphi, b_{\rho}^{\prime}\right] .
$$

Using Eq. (43) and the formula:

$$
\left[\ddot{\varphi}, b_{\rho}^{\prime}\right]=-2 i \tilde{f} \partial_{\rho} \dot{\varphi} \delta^{3}+2 i \tilde{f} \partial_{\rho} \varphi \tilde{g}^{0 k} \partial_{k}\left(\tilde{f} \delta^{3}\right),
$$

which can be proved through (48) and (52), we can show that the rhs of Eq. (A12) is identically vanishing. Accordingly, we have succeeded in proving our statement (A1).

\section{APPENDIX B: CONSERVED CURRENT FOR SCALE SYMMETRY}

In this Appendix, we will present a derivation of the conserved current for the scale symmetry in Eq. (102) and show that the current is indeed conserved on-shell.

First, let us rewrite the Einstein-Hilbert term in order not to include second order derivatives of the metric by the standard technique [30]:

$\sqrt{-g} R=\tilde{g}^{\mu \nu}\left(\partial_{\alpha} \Gamma_{\mu \nu}^{\alpha}-\partial_{\nu} \Gamma_{\mu \alpha}^{\alpha}+\Gamma_{\mu \nu}^{\sigma} \Gamma_{\sigma \alpha}^{\alpha}-\Gamma_{\mu \alpha}^{\sigma} \Gamma_{\nu \sigma}^{\alpha}\right)$,

where the first and second terms can be rewritten as

$$
\begin{aligned}
\tilde{g}^{\mu \nu} \partial_{\alpha} \Gamma_{\mu \nu}^{\alpha} & =\partial_{\alpha}\left(\tilde{g}^{\mu \nu} \Gamma_{\mu \nu}^{\alpha}\right)-\partial_{\alpha} \tilde{g}^{\mu \nu} \cdot \Gamma_{\mu \nu}^{\alpha} \\
& =\partial_{\alpha}\left(\tilde{g}^{\mu \nu} \Gamma_{\mu \nu}^{\alpha}\right)-\left(\Gamma_{\alpha \rho}^{\rho} \tilde{g}^{\mu \nu}-2 \Gamma_{\alpha \rho}^{\mu} \tilde{g}^{\nu \rho}\right) \Gamma_{\mu \nu}^{\alpha} \\
\tilde{g}^{\mu \nu} \partial_{\nu} \Gamma_{\mu \alpha}^{\alpha} & =\partial_{\nu}\left(\tilde{g}^{\mu \nu} \Gamma_{\mu \alpha}^{\alpha}\right)-\partial_{\nu} \tilde{g}^{\mu \nu} \cdot \Gamma_{\mu \alpha}^{\alpha} \\
& =\partial_{\nu}\left(\tilde{g}^{\mu \nu} \Gamma_{\mu \alpha}^{\alpha}\right)+\Gamma_{\rho \sigma}^{\mu} \tilde{g}^{\rho \sigma} \Gamma_{\mu \alpha}^{\alpha} .
\end{aligned}
$$

From Eqs. (B1) and (B2), we have

$$
\begin{aligned}
\sqrt{-g} R= & \partial_{\alpha}\left(\tilde{g}^{\mu \nu} \Gamma_{\mu \nu}^{\alpha}-\tilde{g}^{\mu \alpha} \Gamma_{\mu \beta}^{\beta}\right) \\
& +\tilde{g}^{\mu \nu}\left(\Gamma_{\mu \alpha}^{\sigma} \Gamma_{\nu \sigma}^{\alpha}-\Gamma_{\mu \nu}^{\sigma} \Gamma_{\sigma \alpha}^{\alpha}\right) .
\end{aligned}
$$

Using the formula (B3), up to a surface term the part of the scalar-tensor gravity in the classical Lagrangian (69) can be cast to the form:

$$
\sqrt{-g} \varphi R=-\sqrt{-g}\left(G^{\alpha} \partial_{\alpha} \varphi+G \varphi\right)
$$

where $G^{\alpha}$ and $G$ are defined by

$$
\begin{aligned}
G^{\alpha} & \equiv g^{\mu \nu} \Gamma_{\mu \nu}^{\alpha}-g^{\mu \alpha} \Gamma_{\mu \beta}^{\beta}, \\
G & \equiv g^{\mu \nu}\left(\Gamma_{\mu \nu}^{\sigma} \Gamma_{\sigma \alpha}^{\alpha}-\Gamma_{\mu \alpha}^{\sigma} \Gamma_{\nu \sigma}^{\alpha}\right) .
\end{aligned}
$$

Then, the quantum Lagrangian (70) takes the form:

$$
\begin{aligned}
\mathcal{L}_{q}= & -\sqrt{-g}\left(G^{\alpha} \partial_{\alpha} \varphi+G \varphi+\frac{1}{2} g^{\mu \nu} \partial_{\mu} \phi \partial_{\nu} \phi\right) \\
& -\tilde{g}^{\mu \nu} \partial_{\mu} b_{\nu}-i \tilde{g}^{\mu \nu} \partial_{\mu} \bar{c}_{\rho} \partial_{\nu} c^{\rho} .
\end{aligned}
$$

With the infinitesimal scale invariance (103), the Noether theorem provides us with a formula for the conserved current:

$$
\Lambda J^{\mu}=\sum_{i} \frac{\partial^{R} \mathcal{L}_{q}}{\partial \partial_{\mu} \Phi_{i}} \delta \Phi_{i}
$$

where we use the right-derivative notation and $\Phi_{i}=$ $\left\{g_{\rho \sigma}, \phi, b_{\rho}, \bar{c}_{\rho}, c^{\rho}\right\}$. It is therefore necessary to evaluate each term on the rhs of the following equation:

$$
\begin{aligned}
J^{\mu}= & \frac{\partial^{R} \mathcal{L}_{q}}{\partial \partial_{\mu} g_{\rho \sigma}} 2 g_{\rho \sigma}-\frac{\partial^{R} \mathcal{L}_{q}}{\partial \partial_{\mu} \phi} \phi-\frac{\partial^{R} \mathcal{L}_{q}}{\partial \partial_{\mu} b_{\rho}} 2 b_{\rho} \\
& -\frac{\partial^{R} \mathcal{L}_{q}}{\partial \partial_{\mu} \bar{c}_{\rho}} \bar{c}_{\rho}-\frac{\partial^{R} \mathcal{L}_{q}}{\partial \partial_{\mu} c^{\rho}} c^{\rho} .
\end{aligned}
$$

To calculate the first term on the rhs, we need the formulas:

$$
\begin{aligned}
\frac{\partial\left(\sqrt{-g} G^{\alpha}\right)}{\partial \partial_{\mu} g_{\rho \sigma}}= & \sqrt{-g}\left(g^{\alpha(\rho} g^{\sigma) \mu}-g^{\rho \sigma} g^{\mu \alpha}\right), \\
\frac{\partial(\sqrt{-g} G)}{\partial \partial_{\mu} g_{\rho \sigma}}= & \sqrt{-g}\left[\frac{1}{2} g^{\rho \sigma} g^{\alpha \beta} \Gamma_{\alpha \beta}^{\mu}+\Gamma_{\alpha \lambda}^{\alpha}\left(g^{\lambda(\rho} g^{\sigma) \mu}-\frac{1}{2} g^{\lambda \mu} g^{\rho \sigma}\right)\right. \\
& \left.+\frac{1}{2}\left(g^{\lambda(\rho} \partial_{\lambda} g^{\sigma) \mu}-\frac{1}{2} g^{\lambda \mu} \partial_{\lambda} g^{\rho \sigma}-g^{\rho \alpha} g^{\beta \sigma} \Gamma_{\alpha \beta}^{\mu}\right)\right],
\end{aligned}
$$

where we have used the symmetrization notation, $A_{(\rho} B_{\sigma)} \equiv \frac{1}{2}\left(A_{\rho} B_{\sigma}+A_{\sigma} B_{\rho}\right)$. After a straightforward calculation, we find that the current is given by

$$
\begin{aligned}
J^{\mu} & =\tilde{g}^{\mu \nu}\left[\frac{6 \xi+1}{\xi} \partial_{\nu} \varphi+2 b_{\nu}+i \partial_{\nu}\left(\bar{c}_{\rho} c^{\rho}\right)\right] \\
& =\tilde{g}^{\mu \nu}\left[\frac{6 \xi+1}{2} \partial_{\nu}\left(\phi^{2}\right)+2 b_{\nu}+i \partial_{\nu}\left(\bar{c}_{\rho} c^{\rho}\right)\right],
\end{aligned}
$$

where we have used $\varphi \equiv \frac{1}{2} \xi \phi^{2}$. Note that in the case of the Weyl invariant scalar-tensor gravity where $6 \xi+1=0$ is 
satisfied, the first term, which is a classical contribution, on the rhs in the above equation identically vanishes $[17,18]$.

Finally, let us check that this current is in fact conserved in terms of the field equations (71). Taking the derivative of the former current in (B10) leads to

$$
\partial_{\mu} J^{\mu}=\sqrt{-g}\left(\frac{6 \xi+1}{\xi} \square \varphi+E\right),
$$

where we have used the de Donder gauge and the field equations for the FP ghosts. It is easy to see that this expression vanishes by using the field equations (71). To do so, let us take the trace of the Einstein equation, i.e., the first equation, in Eq. (71) whose result reads

$$
(3 \square-R) \varphi-\frac{1}{2} T^{\mu}{ }_{\mu}+\frac{1}{2} E=0 .
$$

From the field equation for the scalar field, i.e., the second equation, in Eq. (71), we obtain

$$
\varphi R=-\frac{1}{2} \phi \square \phi .
$$

Moreover, taking the trace of $T_{\mu \nu}$ in Eq. (72) gives rise to

$$
T^{\mu}{ }_{\mu}=-\left(\partial_{\rho} \phi\right)^{2} .
$$

Then, substituting Eqs. (B13) and (B14) into (B12), we have

$$
3 \square \varphi+\frac{1}{2}\left[\phi \square \phi+\left(\partial_{\rho} \phi\right)^{2}\right]+\frac{1}{2} E=0 .
$$

Since the definition $\varphi \equiv \frac{1}{2} \xi \phi^{2}$ gives us an equation:

$$
\square \varphi=\xi\left[\phi \square \phi+\left(\partial_{\rho} \phi\right)^{2}\right],
$$

inserting this equation to Eq. (B15) yields the desired equation:

$$
\frac{6 \xi+1}{\xi} \square \varphi+E=0,
$$

by which we can prove the conservation law of the scale current, $\partial_{\mu} J^{\mu}=0$ as seen in Eq. (B11).
[1] J. Polchinski, String Theory (Cambridge University Press, Cambridge, England, 1998).

[2] B. Zumino, Effective Lagrangian and Broken Symmetries, Lectures on Elementary Particles and Quantum Field Theory v.2 (Brandeis University, Cambridge, MA, 1970), pp. 437-500.

[3] Y. Fujii and K. Maeda, The Scalar-Tensor Theory of Gravitation (Cambridge University Press, Cambridge, England, 2003).

[4] I. Oda, Restricted Weyl symmetry, Phys. Rev. D 102, 045008 (2020).

[5] A. Kamimura and I. Oda, Quadratic gravity and restricted Weyl symmetry, Mod. Phys. Lett. A 36, 2150139 (2021).

[6] I. Oda, Restricted Weyl symmetry and spontaneous symmetry breakdown of conformal symmetry, Mod. Phys. Lett. A 36, 2150203 (2021).

[7] N. Nakanishi, Indefinite metric quantum field theory of general gravity, Prog. Theor. Phys. 59, 972 (1978).

[8] N. Nakanishi and I. Ojima, Covariant Operator Formalism of Gauge Theories and Quantum Gravity (World Scientific Publishing, Singapore, 1990), and references therein.

[9] N. Nakanishi and I. Ojima, Proof of the Exact Masslessness of Gravitons, Phys. Rev. Lett. 43, 91 (1979).

[10] T. Kugo and I. Ojima, Local covariant operator formalism of nonabelian gauge theories and quark confinement problem, Prog. Theor. Phys. Suppl. 66, 1 (1979).

[11] C. Brans and R. H. Dicke, Mach's principle and a relativistic theory of gravitation, Phys. Rev. 124, 925 (1961).
[12] C. W. Misner, K. S. Thorne, and J. A. Wheeler, Gravitation (W. H. Freeman and Co (Sd), San Francisco, 1973).

[13] T. Kugo, Noether currents and Maxwell-type equations of motion in higher derivative gravity theories, Symmetry 13, 1408 (2021).

[14] A. Edery and Y. Nakayama, Restricted Weyl invariance in four-dimensional curved spacetime, Phys. Rev. D 90, 043007 (2014).

[15] A. Edery and Y. Nakayama, Generating Einstein gravity, cosmological constant and higgs mass from restricted Weyl invariance, Mod. Phys. Lett. A 30, 1550152 (2015).

[16] A. Edery and Y. Nakayama, Critical gravity from four dimensional scale invariant gravity, J. High Energy Phys. 11 (2019) 169.

[17] R. Jackiw and S-Y. Pi, Fake conformal symmetry in conformal cosmological models, Phys. Rev. D 91, 067501 (2015).

[18] I. Oda, Fake conformal symmetry in unimodular gravity, Phys. Rev. D 94, 044032 (2016).

[19] F. Englert, C. Truffin, and R. Gastmans, Conformal invariance in quantum gravity, Nucl. Phys. 117, 407 (1976).

[20] M. Shaposhnikov and D. Zenhausern, Quantum scale invariance, cosmological constant and hierarchy problem, Phys. Lett. B 671, 162 (2009).

[21] M. Shaposhnikov and F. Tkachov, Quantum scale-invariant models as effective field theories, arXiv:0905.4857.

[22] A. Codello, G. D’Odorico, C. Pagani, and R. Percacci, The renormalization group and Weyl-invariance, Classical Quantum Gravity 30, 115015 (2013). 
[23] C. Tamarit, Running couplings with a vanishing scale anomaly, J. High Energy Phys. 12 (2013) 098.

[24] D. M. Ghilencea, Manifestly scale-invariant regularization and quantum effective operators, Phys. Rev. D 93, 105006 (2016).

[25] I. Oda, Scale invariance and dilaton mass, arXiv: 2110.15408.

[26] I. Oda, Planck and electroweak scales emerging from conformal gravity, Eur. Phys. J. C 78, 798 (2018).
[27] I. Oda, Higgs potential from Weyl conformal gravity, Mod. Phys. Lett. A 35, 2050304 (2020).

[28] I. Oda, Planck scale from broken local conformal invariance in Weyl geometry, Adv. Stud. Theor. Phys. 14, 9 (2020).

[29] C. P. Burgess and F. Quevedo, Axion homeopathy: Screening dilaton interactions, arXiv:2110.10352.

[30] L. D. Landau and E. M. Lifshitz, The Classical Theory of Fields, Course of Theoretical Physics Series Vol. 2 (Butterworth-Heinemann, London, 1980). 